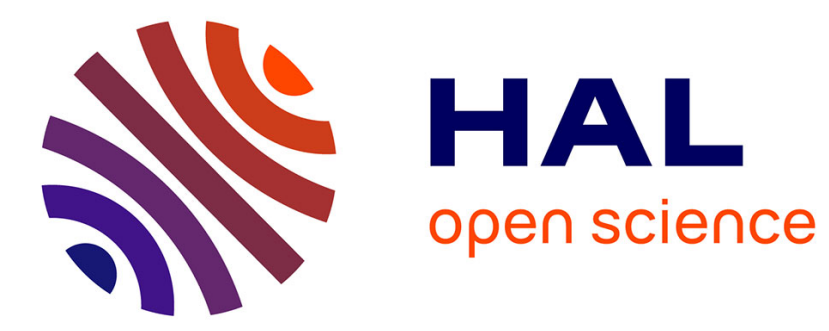

\title{
DIFFERENTIABLE RIGIDITY UNDER RICCI CURVATURE LOWER BOUND
}

Laurent Bessières, Gérard Besson, Gilles Courtois, Sylvestre Gallot

\section{To cite this version:}

Laurent Bessières, Gérard Besson, Gilles Courtois, Sylvestre Gallot. DIFFERENTIABLE RIGIDITY UNDER RICCI CURVATURE LOWER BOUND. Duke Mathematical Journal, 2012, 161 (1), pp.2967. 10.1215/00127094-1507272 . hal-00281855v3

\section{HAL Id: hal-00281855 \\ https://hal.science/hal-00281855v3}

Submitted on 13 Feb 2020

HAL is a multi-disciplinary open access archive for the deposit and dissemination of scientific research documents, whether they are published or not. The documents may come from teaching and research institutions in France or abroad, or from public or private research centers.
L'archive ouverte pluridisciplinaire HAL, est destinée au dépôt et à la diffusion de documents scientifiques de niveau recherche, publiés ou non, émanant des établissements d'enseignement et de recherche français ou étrangers, des laboratoires publics ou privés. 


\title{
DIFFERENTIABLE RIGIDITY UNDER RICCI CURVATURE LOWER BOUND
}

L. BESSIÈRES, G. BESSON, G. COURTOIS, and S. GALLOT

\begin{abstract}
In this article we prove a differentiable rigidity result. Let $(Y, g)$ and $\left(X, g_{0}\right)$ be two closed n-dimensional Riemannian manifolds $(n \geq 3)$, and let $f: Y \rightarrow X$ be a continuous map of degree 1 . We furthermore assume that the metric $g_{0}$ is real hyperbolic and denote by $d$ the diameter of $\left(X, g_{0}\right)$. We show that there exists a number $\varepsilon:=\varepsilon(n, d)>0$ such that if the Ricci curvature of the metric $g$ is bounded below by $-(n-1) g$ and its volume satisfies $\operatorname{vol}_{g}(Y) \leq(1+\varepsilon) \operatorname{vol}_{g_{0}}(X)$, then the manifolds are diffeomorphic. The proof relies on Cheeger-Colding's theory of limits of Riemannian manifolds under lower Ricci curvature bound.
\end{abstract}

\section{Introduction}

Let $Y$ and $X$ be two closed manifolds. The manifold $Y$ is said to dominate $X$ if there is a continuous map $f: Y \rightarrow X$ of degree one. An $n$-dimensional hyperbolic manifold $X$ has the smallest volume among the set of all Riemannian manifolds $(Y, g)$ such that $Y$ dominates $X$ and the metric $g$ has Ricci curvature $\operatorname{Ric}_{g} \geq-(n-1) g$. In dimension $n=2$ this is a consequence of the Gauss-Bonnet formula, and in dimension $n \geq 3$ this follows from the following.

THEOREM 1.1 ([3, p. 734])

Let $\left(X, g_{0}\right)$ be an $n$-dimensional closed hyperbolic manifold, and let $Y$ be a closed manifold that dominates $X$. Then, for any metric $g$ on $Y$ such that $\operatorname{Ric}_{g} \geq-(n-1) g$, one has $\operatorname{vol}_{g}(Y) \geq \operatorname{vol}_{g_{0}}(X)$, and equality happens if and only if $(Y, g)$ and $\left(X, g_{0}\right)$ are isometric.

The minimal volume of a closed manifold $Y$ is defined as

$$
\operatorname{minvol}(Y)=\inf \left\{\operatorname{vol}_{g}(Y) ;\left|\mathrm{K}_{g}\right| \leq 1\right\}
$$

DUKE MATHEMATICAL JOURNAL

Vol. 161, No. 1, () 2012 DOI 10.1215/00127094-1507272

Received 17 February 2010. Revision received 17 June 2011.

2010 Mathematics Subject Classification. Primary 53C20; Secondary 53C35.

Authors' work partially supported by Agence Nationale de la Recherche grant ANR-07-BLAN-0251. 
where $\mathrm{K}_{g}$ is the sectional curvature of the Riemannian metric $g$. An $n$-dimensional hyperbolic manifold $X$ is characterized by its minimal volume among the set of all Riemannian manifolds $Y$ such that $Y$ is homotopy equivalent to $X$. Namely, we have the following.

\section{THEOREM 1.2 ([2, Theorem 1.1])}

Let $X$ be an n-dimensional closed hyperbolic manifold, and let $Y$ be a closed manifold that dominates $X$. Then, $\operatorname{minvol}(Y)=\operatorname{minvol}(X)$ if and only if $X$ and $Y$ are diffeomorphic.

The aim of this paper is to show the following gap result. It improves the above Theorem 1.2 since we now require a lower bound on the Ricci curvature instead of a pinching of the sectional curvature; moreover, under the hypothesis, we prove that if the volume of $Y$ is close to the volume of $X$, then these two manifolds are diffeomorphic. More precisely, we have the following.

\section{THEOREM 1.3}

Given any integer $n \geq 3$ and $d>0$, there exists $\varepsilon(n, d)>0$ such that the following holds. Suppose that $\left(X, g_{0}\right)$ is an n-dimensional closed hyperbolic manifold with diameter $\leq d$ and that $Y$ is a closed manifold that dominates $X$; that is, there exists a degree-one map $f: Y \rightarrow X$. Then $Y$ has a metric $g$ such that

$$
\begin{aligned}
\operatorname{Ric}_{g} & \geq-(n-1) g, \\
\operatorname{vol}_{g}(Y) & \leq(1+\varepsilon) \operatorname{vol}_{g_{0}}(X)
\end{aligned}
$$

if and only if $f$ is homotopic to a diffeomorphism.

In [10] the authors prove the existence of closed $n$-dimensional manifolds $Y$ that are homeomorphic to a closed $n$-dimensional hyperbolic manifold $\left(X, g_{0}\right)$ but not diffeomorphic to it. An immediate corollary of the above theorem is the following.

\section{COROLLARY 1.4}

With the above notation, there exists $\varepsilon>0$ depending on $n$ and on the diameter of $X$ with the property that for any such $Y$ and any Riemannian metric $g$ on $Y$ whose Ricci curvature is bounded below by $-(n-1)$ one has

$$
\operatorname{vol}(Y, g)>(1+\varepsilon) \operatorname{vol}\left(X, g_{0}\right) .
$$

To be more precise, in [10] the manifold $Y$ is obtained as follows:

$$
Y=X \sharp \Sigma,
$$


where $\Sigma$ is an exotic sphere. Not every closed hyperbolic manifold $X$ gives rise to such a $Y$ that is (obviously) homeomorphic but not diffeomorphic to $X$. Indeed, we may have to take a finite cover of $X$. But when we get one construction that works, it does on any finite cover $\bar{X}$ of $X$ as well. The authors also prove that by taking covers of arbitrary large degree we can put on $Y$ a metric whose sectional curvature is arbitrarily pinched around, say, -1 . The stronger the pinching, the larger the degree. Now assume that $\varepsilon$ could be taken independent of the diameter of $X$; applying the results of [3] one could show that the volumes of the two manifolds are very close when the pinching on $Y$ is very sharp (close to -1 ). The volume of $Y$ endowed with this pinched metric could then be taken smaller than $(1+\varepsilon) \operatorname{vol}\left(X, g_{0}\right)$, by choosing a covering of large degree; the manifolds, though, are not diffeomorphic. This gives a contradiction and shows that "size" of $X$ has to be involved in the statement of the theorem, for example, its diameter.

\subsection{Sketch of the proof}

We argue by contradiction. Suppose that there is a sequence $\left(X_{k}\right)_{k \in \mathbf{N}}$ of closed hyperbolic manifolds with diameter $\leq d$ and a sequence of closed manifolds $Y_{k}$, of degreeone continuous maps $f_{k}: Y_{k} \rightarrow X_{k}$ and metrics $g_{k}$ on $Y_{k}$ satisfying hypotheses (3) and (4) for some $\varepsilon_{k}$ going to zero. Since $f_{k}$ is of degree one and $X_{k}$ is hyperbolic, it is equivalent to say (thanks to Mostow's rigidity theorem) that $f_{k}$ is homotopic to a diffeomorphism or simply that $X_{k}$ and $Y_{k}$ are diffeomorphic. We thus assume that $Y_{k}$ and $X_{k}$ are not diffeomorphic. One then shows that up to a subsequence, for large $k$, $Y_{k}$ is diffeomorphic to a closed manifold $Y, X_{k}$ is diffeomorphic to a closed manifold $X$, and $X$ and $Y$ are diffeomorphic. One argues as follows: by the classical finiteness results we get the subconvergence of the sequence $\left\{X_{k}\right\}$. Indeed, the curvature is -1 , the diameter is bounded by hypothesis, and there is a universal lower bound for the volume of any closed hyperbolic manifold of a given dimension, thanks to Margulis's lemma (see [6, Theorem 37.1.1]). Cheeger's finiteness theorem then applies. Moreover, on a closed manifold of dimension $\geq 3$, there is at most one hyperbolic metric, up to isometry. We can therefore suppose that $X_{k}=X$ is a fixed hyperbolic manifold. The inequality proved in Theorem 1.1 provides a lower bound for the volume of $Y_{k}$ as it is explained below. We have no a priori bounds on the diameter of $\left(Y_{k}, g_{k}\right)$, but we can use Cheeger-Colding's theory to obtain subconvergence in the pointed GromovHausdorff topology to a complete metric space $(Z, d)$ with small singular set. To obtain more geometric control, the idea is to use the natural maps between $Y_{k}$ and $X$ (see [3]). One can show that they subconverge to a limit map between $Z$ and $X$, which is an isometry. Then $X$ is an $n$-dimensional smooth closed Riemannian manifold, which is the Gromov-Hausdorff limit of the sequence $\left(Y_{k}, g_{k}\right)$ of a Riemannian 
manifold of dimension $n$ satisfying the lower bound (3) on Ricci curvature; therefore $X$ and $Y_{k}$ are diffeomorphic for large $k$ by a theorem of J. Cheeger and T. Colding.

The paper is organized as follows. The construction and the properties of the natural maps are given in Section 3. In Section 4, we construct the limit space $Z$ and the limit map $F: Z \rightarrow X$. In Section 5, we prove that $F$ is an isometry and conclude.

\subsection{Maps of arbitrary degree and scalar curvature}

For two closed manifolds $Y$ and $X$ we said above that $Y$ dominates $X$ if there exists a map of degree one from $Y$ onto $X$. We could have required that there exist a map $f: Y \rightarrow X$ of nonzero degree. The main theorem of [3] was stated and proved in this setup. More precisely, the following statement holds.

\section{THEOREM 1.5 (see [3])}

Let $\left(X, g_{0}\right)$ be an $n$-dimensional closed hyperbolic manifold, and let $Y$ be a closed manifold such that there exists a map $f: Y \rightarrow X$ with nonzero degree denoted $\operatorname{deg}(f)$. Then, for any metric $g$ on $Y$ such that $\operatorname{Ric}_{g} \geq-(n-1) g$, one has $\operatorname{vol}_{g}(Y) \geq$ $|\operatorname{deg}(f)| \operatorname{vol}_{g_{0}}(X)$, and equality happens if and only if $f$ is homotopic to a Riemannian covering (i.e., locally isometric) of degree $|\operatorname{deg}(f)|$ from $(Y, g)$ onto $\left(X, g_{0}\right)$.

With the technique developed in this article, the following result can be proved.

\section{THEOREM 1.6}

Given any integer $n \geq 3$ and $d>0$, there exists $\varepsilon(n, d)>0$ such that the following holds. Suppose that $\left(X, g_{0}\right)$ is an n-dimensional closed hyperbolic manifold with diameter $\leq d$ and that $Y$ is a closed manifold such that there exists a map $f: Y \rightarrow X$ with nonzero degree. Then $Y$ has a metric $g$ such that

$$
\begin{aligned}
\operatorname{Ric}_{g} & \geq-(n-1) g \\
\operatorname{vol}_{g}(Y) & \leq(1+\varepsilon)|\operatorname{deg}(f)| \operatorname{vol}_{g_{0}}(X)
\end{aligned}
$$

if and only if $f$ is homotopic to a covering of degree $|\operatorname{deg}(f)|$.

The proof is essentially the one described above; it uses the technique described in the remainder of this text and the treatment of an arbitrary degree given in [2]. The fact that the degree can be, in absolute value, greater than one yields extra technicalities. For the sake of clarity we shall omit this proof in the present article and leave it to the reader. A corollary is the following.

COROLLARY 1.7

Let $\left(X, g_{0}\right)$ be a closed $n$-dimensional hyperbolic manifold. Then there exists $\varepsilon>$ 
0 , such that, for any metric $g$ on the connected sum $X \sharp X$ satisfying that its Ricci curvature of $g$ is not smaller than $-(n-1)$,

$$
\operatorname{vol}(X \sharp X, g) \geq 2(1+\varepsilon) \operatorname{vol}\left(X, g_{0}\right) .
$$

Indeed, $X \sharp X$ does not carry a hyperbolic metric and hence is not a double cover of $X$. This can be proven, for example, by saying that the $(n-1)$-sphere on which the connected sum is made does not bound a topological $n$-ball, whereas in a hyperbolic manifold every such sphere bounds a ball. We may now ask whether such a result could be true with a lower bound on the scalar curvature instead of a lower bound on the Ricci curvature. The situation in dimension 3, completely clarified by Perelman's work, shows that the answer to this question is negative. More precisely, if $\left(X, g_{0}\right)$ is a 3-dimensional closed hyperbolic manifold, a consequence of [1, inequality 2.10$]$ is that

$$
\inf \{\operatorname{vol}(X \sharp X, g) ; \operatorname{Scal}(g) \geq-6\}=2 \operatorname{vol}\left(X, g_{0}\right) .
$$

In dimensions greater than or equal to 4, it follows from [15] and the solution to the Yamabe problem that

$$
\inf \{\operatorname{vol}(X \sharp X, g) ; \operatorname{Scal}(g) \geq-6\} \leq 2 \operatorname{vol}\left(X, g_{0}\right) .
$$

\section{Some a priori control on $(Y, g)$}

Some a priori control on the metric $g$ will be needed in Sections 2 and 3. We give here the necessary results.

Let $\left(X, g_{0}\right)$ be a hyperbolic manifold, and let $Y$ be a manifold satisfying the assumptions of Theorem 1.3. For any Riemannian metric $g$ on $Y$ satisfying the curvature assumption (3), one has the following inequality:

$$
\operatorname{vol}_{g}(Y) \geq \operatorname{vol}_{g_{0}}(X) .
$$

It is a consequence of Besson-Courtois-Gallot's inequality (see [3])

$$
h(g)^{n} \operatorname{vol}_{g}(Y) \geq h\left(g_{0}\right)^{n} \operatorname{vol}_{g_{0}}(X),
$$

where $h(g)$ is the volume entropy, or the critical exponent, of the metric $g$, that is,

$$
h(g)=\lim _{R \rightarrow+\infty} \frac{1}{R} \ln \left(\operatorname{vol}_{\tilde{g}}\left(B_{\tilde{g}}(x, R)\right)\right),
$$

where $\tilde{g}$ is the lifted metric on $\tilde{Y}$. Indeed, any metric $g$ on $Y$ which satisfies (3) verifies, by Bishop's theorem,

$$
h(g) \leq h\left(g_{0}\right)=n-1 .
$$


One can obtain a lower bound of the volume of some balls by Gromov's isolation theorem (see [13, Theorem 0.5]). It shows that if the simplicial volume $\|Y\|-$ a topological invariant also called Gromov's norm — of $Y$ is nonzero, then for any Riemannian metric $g$ on $Y$ satisfying the curvature assumption (3), there exists at least one point $y_{g} \in Y$ such that

$$
\operatorname{vol}_{g}\left(B\left(y_{g}, 1\right)\right) \geq v_{n}>0 .
$$

Here $B\left(y_{g}, 1\right)$ is the geodesic ball of radius 1 for the metric $g$, and $v_{n}$ is a universal constant. This theorem applies in our situation since, by an elementary property of the simplicial volume, $\|Y\| \geq\|X\|$ if there is a degree-one map from $Y$ to $X$ (see [13]). On the other hand, $X$ has a hyperbolic metric, and hence $\|X\|>0$ by GromovThurston's theorem (see [13]).

Given this universal lower bound for the volume of a unit ball $B\left(y_{g}, 1\right)$, the volume of any ball $B(y, r)$ is bounded from below in terms of $r$ and $d\left(y_{g}, y\right)$. Indeed, recall that under the curvature assumption (3), Bishop-Gromov's theorem shows that for any $0<r \leq R$, one has

$$
\frac{\operatorname{vol}_{g}(B(y, r))}{\operatorname{vol}_{g}(B(y, R))} \geq \frac{\operatorname{vol}_{\mathbf{H}^{n}}\left(\mathrm{~B}_{\mathbf{H}^{n}}(r)\right)}{\left.\operatorname{vol}_{\mathbf{H}^{n}}\left(\mathrm{~B}_{\mathbf{H}^{n}} R\right)\right)},
$$

where $\mathrm{B}_{\mathbf{H}^{n}}(r)$ is a ball of radius $r$ in the hyperbolic space $\mathbf{H}^{n}$. As $B\left(y_{g}, 1\right) \subset B(y, 1+$ $\left.d\left(y_{g}, y\right)+r\right)$, one deduces from (9) that

$$
\begin{aligned}
\operatorname{vol}_{g} & (B(y, r)) \\
& \geq \operatorname{vol}_{g}\left(B\left(y, 1+d\left(y_{g}, y\right)+r\right)\right) \frac{\operatorname{vol}_{\mathbf{H}^{n}}\left(\mathrm{~B}_{\mathbf{H}^{n}}(r)\right)}{\operatorname{vol}_{\mathbf{H}^{n}}\left(\mathrm{~B}_{\mathbf{H}^{n}}\left(1+d\left(y_{g}, y\right)+r\right)\right)} \\
& \geq v_{n} \frac{\operatorname{vol}_{\mathbf{H}^{n}}\left(\mathrm{~B}_{\mathbf{H}^{n}}(r)\right)}{\operatorname{vol}_{\mathbf{H}^{n}}\left(\mathrm{~B}_{\mathbf{H}^{n}}\left(1+d\left(y_{g}, y\right)+r\right)\right)} .
\end{aligned}
$$

The curvature assumption (3) and the volume estimates (9) or (11) are those required to use the noncollapsing part of Cheeger-Colding's theory, as we shall see in Section 3.

\section{The natural maps}

In Sections 3.1 and 3.2 we recall the construction and the main properties of the natural maps defined in [3] (see also [4]).

\subsection{Construction of the natural maps}

Suppose that $(Y, g)$ and $\left(X, g_{0}\right)$ are closed Riemannian manifolds and that

$$
f: Y \rightarrow X
$$


is a continuous map of degree one. For the sake of simplicity, we assume that $g_{0}$ is hyperbolic. (The construction holds in a much more general situation.) Then, for any $c>h(g)$ there exists a $C^{1}$-map

$$
F_{c}: Y \longrightarrow X
$$

homotopic to $f$, such that for all $y \in Y$,

$$
\left|\operatorname{Jac} F_{c}(y)\right| \leq\left(\frac{c}{h\left(g_{0}\right)}\right)^{n},
$$

with equality for some $y \in Y$ if and only if $d_{y} F_{c}$ is a homothety of ratio $\frac{c}{h\left(g_{0}\right)}$.

Inequality (6) is then easily obtained by integration of (12) and by taking a limit when $c$ goes to $h(g)$. In general, to obtain global rigidity properties, one has to study carefully the behavior of $F_{c}$ as $c$ goes to $h(g)$.

The construction of the maps is divided in four steps. Let $\tilde{Y}$ and $\tilde{X}$ be the universal coverings of $Y$ and $X$, respectively, and let $\tilde{f}: \tilde{Y} \rightarrow \tilde{X}$ be a lift of $f$.

Step 1. For each $y \in \tilde{Y}$ and $c>h(g)$, let $v_{y}^{c}$ be the finite measure on $\tilde{Y}$ defined by

$$
d \nu_{y}^{c}(z)=e^{-c . \rho(y, z)} \mathrm{dv}_{\tilde{\mathrm{g}}}(z)
$$

where $z \in \tilde{Y}, \tilde{g}$ is the lifted metric on $\tilde{Y}$ and $\rho(.,$.$) is the distance function of (\tilde{Y}, \tilde{g})$.

Step 2. Pushing forward this measure gives a finite measure $\tilde{f}_{*} v_{y}^{c}$ on $\tilde{X}$. Let us recall that it is defined by

$$
\tilde{f}_{*} v_{y}^{c}(U)=v_{y}^{c}\left(\tilde{f}^{-1}(U)\right) .
$$

Step 3. One defines a finite measure $\mu_{y}^{c}$ on $\partial \tilde{X}$ by convolution of $\tilde{f}_{*} \nu_{y}^{c}$ with all visual probability measures $P_{x}$ of $\tilde{X}$. Recall that the visual probability measure $P_{x}$ at $x \in \tilde{X}$ is defined as follows: the unit tangent sphere at $x$ noted $U_{x} \tilde{X}$ projects onto the geometric boundary $\partial \tilde{X}$ by the map

$$
v \in U_{x} \tilde{X} \stackrel{E_{x}}{\longrightarrow} \gamma_{v}(\infty) \in \partial \tilde{X},
$$

where $\gamma_{v}(t)=\exp _{x}(t v)$. The measure $P_{x}$ is then the pushforward by $E_{x}$ of the canonical probability measure on $U_{x} \tilde{X}$; that is, for a Borel set $A \in \partial \tilde{X}, P_{x}(A)$ is the measure of the set of vectors $v \in U_{x} \tilde{X}$ such that $\gamma_{v}(+\infty) \in A$.

Then

$$
\mu_{y}^{c}(A)=\int_{\tilde{X}} P_{x}(A) d \tilde{f}_{*} \nu_{y}^{c}(x)=\int_{\tilde{Y}} P_{\tilde{f}(z)}(A) d \nu_{y}^{c}(z) .
$$

One can identify $\partial \tilde{X}$ with the unit sphere in $\mathbf{R}^{n}$, by choosing an origin $o \in \tilde{X}$ and using $E_{0}$. The density of this measure is given by (see [3]) 


$$
d \mu_{y}^{c}(\theta)=\left(\int_{\tilde{Y}} e^{-h\left(g_{0}\right) B(\tilde{f}(z), \theta)} e^{-c \rho(y, z)} \operatorname{dv}_{\tilde{\mathrm{g}}}(z)\right) d \theta
$$

where $\theta \in \partial \tilde{X}, d \theta$ is the canonical probability measure on $S^{n-1}$, and $B(., \theta)$ is a Busemann function on $\tilde{X}$ normalized to vanish at $x=o$. We will use the notation

$$
p(x, \theta)=e^{-h\left(g_{0}\right) B(x, \theta)} .
$$

Step 4. The map

$$
F_{c}: \tilde{Y} \longrightarrow \tilde{X}
$$

associates to any $y \in \tilde{Y}$ the unique $x \in \tilde{X}$, which minimizes on $\tilde{X}$ the function

$$
x \rightarrow \mathscr{B}(x)=\int_{\partial \tilde{X}} B(x, \theta) d \mu_{y}^{c}(\theta)
$$

(see [3, Appendix A]).

The maps $F_{c}$ are shown to be $\mathcal{C}^{1}$ and equivariant with respect to the actions of the fundamental groups of $Y$ and $X$ on their respective universal covers. The quotient maps, which are also denoted by $F_{c}: Y \rightarrow X$, are homotopic to $f$. Note that $F_{c}$ depends heavily on the metric $g$.

\subsection{Some technical lemmas}

Let us give some definitions.

\section{Definition 3.1}

For $y \in \tilde{Y}$, let $\sigma_{y}^{c}$ be the probability measure on $\partial \tilde{X}$ defined by

$$
\sigma_{y}^{c}=\frac{\mu_{y}^{c}}{\mu_{y}^{c}(\partial \tilde{X})}
$$

Let us remark that we have

$$
\left\|\mu_{y}^{c}\right\|=\mu_{y}^{c}(\partial \tilde{X})=\int_{\tilde{Y}} e^{-c \rho(y, z)} \mathrm{dv}_{\tilde{\mathrm{g}}}(z)=\left\|\nu_{y}^{c}\right\| .
$$

We consider two positive definite bilinear forms of trace equal to one and the corresponding symmetric endomorphisms.

\section{Definition 3.2}

For any $y \in \tilde{Y}, u, v \in T_{F_{c}(y)} \tilde{X}$,

$$
h_{y}^{c}(u, v)=\int_{\partial \tilde{X}} d B_{\left(F_{c}(y), \theta\right)}(u) d B_{\left(F_{c}(y), \theta\right)}(v) d \sigma_{y}^{c}(\theta)=g_{0}\left(H_{y}^{c}(u), v\right) .
$$


And, for any $y \in \tilde{Y}, u, v \in T_{y} \tilde{Y}$,

$$
h_{y}^{\prime c}(u, v)=\frac{1}{\mu_{y}^{c}(\partial \tilde{X})} \int_{\tilde{Y}} d \rho_{(y, z)}(u) d \rho_{(y, z)}(v) d \nu_{y}^{c}(z)=g\left(H_{y}^{\prime c}(u), v\right) .
$$

\section{LEMMA 3.3}

For any $y \in \tilde{Y}, u \in T_{y} \tilde{Y}, v \in T_{F(y)} \tilde{X}$, one has

$$
\left|g_{0}\left(\left(I-H_{y}^{c}\right) d_{y} F_{c}(u), v\right)\right| \leq c\left(g_{0}\left(H_{y}^{c}(v), v\right)\right)^{1 / 2}\left(g\left(H_{y}^{\prime c}(u), u\right)\right)^{1 / 2} .
$$

Proof

Since $F_{c}(y)$ is an extremum of the function $\mathcal{B}$, one has

$$
d_{F_{c}(y)} \mathcal{B}(v)=\int_{\partial \tilde{X}} d B_{\left(F_{c}(y), \theta\right)}(v) d \mu_{y}^{c}(\theta)=0
$$

for each $v \in T_{F_{c}(y)} \tilde{X}$. By differentiating this equation in a direction $u \in T_{y} \tilde{Y}$, one obtains

$$
\begin{aligned}
\int_{\partial \tilde{X}} & D d B_{\left(F_{c}(y), \theta\right)}\left(d_{y} F_{c}(u), v\right) d \mu_{y}^{c}(\theta) \\
& +\int_{\partial \tilde{X}} d B_{\left(F_{c}(y), \theta\right)}(v)\left(\int_{\tilde{Y}} p(\tilde{f}(z), \theta)\left(-c d \rho_{(y, z)}(u)\right) d \nu_{y}^{c}(z)\right) d \theta=0 .
\end{aligned}
$$

Using Cauchy-Schwarz inequality in the second term, one gets

$$
\begin{aligned}
\mid \int_{\partial \tilde{X}} & D d B_{\left(F_{c}(y), \theta\right)}\left(d_{y} F_{c}(u), v\right) d \mu_{y}^{c}(\theta) \mid \\
\leq & \int_{\partial \tilde{X}}\left|d B_{\left(F_{c}(y), \theta\right)}(v)\right|\left(\int_{\tilde{Y}} p(\tilde{f}(z), \theta) d \nu_{y}^{c}(z)\right)^{1 / 2} \\
& \times\left(\int_{\tilde{Y}} p(\tilde{f}(z), \theta)\left|c d \rho_{(y, z)}(u)\right|^{2} d \nu_{y}^{c}(z)\right)^{1 / 2} d \theta,
\end{aligned}
$$

which is, using Cauchy-Schwarz inequality again,

$$
\begin{aligned}
\leq & c\left(\int_{\partial \tilde{X}}\left|d B_{\left(F_{c}(y), \theta\right)}(v)\right|^{2} \int_{\tilde{Y}} p(\tilde{f}(z), \theta) d \nu_{y}^{c}(z) d \theta\right)^{1 / 2} \\
& \times\left(\int_{\partial \tilde{X}} \int_{\tilde{Y}} p(\tilde{f}(z), \theta)\left|d \rho_{(y, z)}(u)\right|^{2} d \nu_{y}^{c}(z) d \theta\right)^{1 / 2} \\
= & c\left(\int_{\partial \tilde{X}}\left|d B_{\left(F_{c}(y), \theta\right)}(v)\right|^{2} d \mu_{y}^{c}(\theta)\right)^{1 / 2}\left(\int_{\tilde{Y}}\left|d \rho_{(y, z)}(u)\right|^{2} d \nu_{y}^{c}(z)\right)^{1 / 2} \\
= & c \mu_{y}^{c}(\partial \tilde{X})\left(g_{0}\left(H_{y}^{c}(v), v\right)\right)^{1 / 2}\left(g\left(H_{y}^{\prime c}(u), u\right)\right)^{1 / 2} .
\end{aligned}
$$


It is shown in [3, Chapter 5] that $D d B=g_{0}-d B \otimes d B$ for a hyperbolic metric. The left term of the inequality is thus $\mu_{y}^{c}(\partial \tilde{X}) g_{0}\left(\left(I-H_{y}^{c}\right) d_{y} F_{c}(u), v\right)$. This proves the lemma.

\section{Definition 3.4}

Let $0<\lambda_{1}^{c}(y) \leq \cdots \leq \lambda_{n}^{c}(y)<1$ be the eigenvalues of $H_{y}^{c}$.

PROPOSITION 3.5

There exists a constant $A:=A(n)>0$ such that, for any $y \in Y$,

$$
\left|\operatorname{Jac} F_{c}(y)\right| \leq\left(\frac{c}{h\left(g_{0}\right)}\right)^{n}\left(1-A \sum_{i=1}^{n}\left(\lambda_{i}^{c}(y)-\frac{1}{n}\right)^{2}\right) .
$$

\section{Proof}

The proof is based on the two following lemmas.

LEMMA 3.6

At each $y \in \tilde{Y}$,

$$
\left|\operatorname{Jac} F_{c}(y)\right| \leq\left(\frac{c}{\sqrt{n}}\right)^{n} \frac{\operatorname{det}\left(H_{y}^{c}\right)^{1 / 2}}{\operatorname{det}\left(I-H_{y}{ }^{c}\right)} .
$$

\section{Proof}

Let $\left\{v_{i}\right\}$ be an orthonormal basis of $T_{F_{c}(y)} \tilde{X}$ which diagonalizes $H_{y}{ }^{c}$. We can assume that $d_{y} F_{c}$ is invertible since otherwise the above inequality is obvious. Let $u_{i}^{\prime}=[(I-$ $\left.\left.H_{y}{ }^{c}\right) \circ d_{y} F_{c}\right]^{-1}\left(v_{i}\right)$. The Schmidt orthonormalization process applied to $\left(u_{i}^{\prime}\right)$ gives an orthonormal basis $\left(u_{i}\right)$ at $T_{y} \tilde{Y}$. The matrix of $\left(I-H_{y}{ }^{c}\right) \circ d_{y} F_{c}$ in the basis $\left(u_{i}\right)$ and $\left(v_{i}\right)$ is upper triangular; then

$$
\operatorname{det}\left(I-H_{y}{ }^{c}\right) \operatorname{Jac} F_{c}(y)=\prod_{i=1}^{n} g_{0}\left(\left(I-H_{y}{ }^{c}\right) \circ d_{y} F_{c}\left(u_{i}\right), v_{i}\right),
$$

which gives, with (13),

$$
\begin{aligned}
\operatorname{det}\left(I-H_{y}{ }^{c}\right)\left|\operatorname{Jac} F_{c}(y)\right| & \leq c^{n}\left(\prod_{i=1}^{n} g_{0}\left(H_{y}{ }^{c}\left(v_{i}\right), v_{i}\right)\right)^{1 / 2}\left(\prod_{i=1}^{n} g\left(H_{y}^{\prime c}\left(u_{i}\right), u_{i}\right)\right)^{1 / 2} \\
& \leq c^{n} \operatorname{det}\left(H_{y}{ }^{c}\right)^{1 / 2}\left[\frac{1}{n} \sum_{i=1}^{n} g\left(H_{y}^{\prime c}\left(u_{i}\right), u_{i}\right)\right]^{n / 2} .
\end{aligned}
$$

This proves the desired inequality since $\operatorname{trace}\left(H_{y}^{\prime c}\right)=1$. 
LEMMA 3.7

Let $H$ be a symmetric positive definite $(n \times n)$-matrix whose trace is equal to one. Then, if $n \geq 3$,

$$
\frac{\operatorname{det}(H)^{1 / 2}}{\operatorname{det}(I-H)} \leq\left(\frac{n}{h\left(g_{0}\right)^{2}}\right)^{n / 2}\left(1-A \sum_{i=1}^{n}\left(\lambda_{i}-\frac{1}{n}\right)^{2}\right)
$$

for some positive constant $A(n)$.

\section{Proof}

The proof is given in [3, Appendix B5]. This is the point where the rigidity of the natural maps fails in dimension 2 .

This completes the proof of Proposition 3.5.

\subsection{Some nice properties}

We now show that when the volumes of $(Y, g)$ and $\left(X, g_{0}\right)$ are close, the natural maps $F_{c}$ have nice properties. More precisely, we will show that when $\operatorname{vol}(Y, g) \leq$ $(1+\varepsilon) \operatorname{vol}\left(X, g_{0}\right)$ and $0 \leq c-h(g) \leq \delta$ with $\varepsilon$ and $\delta$ small enough, then $d F_{c}$ is almost isometric on a set of large relative volume (see Lemma 3.11). We then prove that $F_{c}$ is uniformly Lipschitz on balls of radius $R$ when $\varepsilon \leq \varepsilon(R)$ and $\delta \leq \delta(R)$ are small enough (see Lemma 3.12). We finally end Section 3 by showing that $F_{c}$ is "quasi-contracting" on balls of radius $R$ (see Lemma 3.13).

In this section, we shall consider $F_{c}$ as a map from $(Y, g)$ to $\left(X, g_{0}\right)$. We suppose that the metric $g$ satisfies the curvature assumption (3) and the assumption on its volume (4) for some $\varepsilon>0$. Let us introduce some terminology.

\section{Definition 3.8}

Let $0<\alpha<1$. We say that a property holds $\alpha$-a.e. ( $\alpha$-almost everywhere) on a set $A$ if the set $A_{+}$of points of $A$ where the property holds has relative volume bigger than or equal to $1-\alpha$, that is,

$$
\frac{\operatorname{vol}\left(A_{+}\right)}{\operatorname{vol}(A)} \geq 1-\alpha
$$

We show that $d F_{c}$ is $\alpha$-close to being isometric $\alpha$-a.e. on $Y$ for some positive $\alpha(\varepsilon, c)$. Moreover, $\alpha(\varepsilon, c) \rightarrow 0$ as $\varepsilon \rightarrow 0$ and $c \rightarrow h(g)$. On the other hand, given any radius $R>0$, one shows that $\left\|d F_{c}\right\|$ is uniformly bounded on balls $B\left(y_{g}, R\right)$, provided $c$ is close enough to $h(g)$. Recall that we have a lower bound for the volume of $(Y, g)$ but we do not have an upper bound for its diameter. The key point is to show 
that $H_{y}^{c}$ is $\alpha$-close to $\frac{1}{n} \mathrm{Id}$ on a set of large volume, and is bounded on a ball of fixed radius, with respect to the parameters $\varepsilon, c$.

To estimate from above $c-h(g)$ we introduce a parameter $\delta>0$. We suppose that the volume entropy of $g$ satisfies the inequalities

$$
h(g)<c \leq h(g)+\delta .
$$

Observe that (7), (15), and (16) imply that

$$
\left|\operatorname{Jac} F_{c}(y)\right| \leq\left(\frac{h(g)+\delta}{h\left(g_{0}\right)}\right)^{n} \leq\left(1+\frac{\delta}{n-1}\right)^{n},
$$

for all $y \in Y$. The map $F_{c}$ is thus almost volume decreasing. On the other hand, as $\operatorname{vol}_{g}(Y)$ is close to $\operatorname{vol}_{g_{0}}(X)$, the set in $Y$ where $F_{c}$ decreases the volume a lot must have a small measure. Equivalently, $\left|\mathrm{Jac} F_{c}\right|$ must be close to 1 in the $L^{1}$-norm. We now give a precise statement.

LEMMA 3.9

If $\delta$ is small enough, there exists $\alpha_{1}=\alpha_{1}(\varepsilon, \delta)>0$ such that for $\alpha_{1}$-a.e. on $Y$ one has,

$$
1-\alpha_{1} \leq\left|\operatorname{Jac} F_{c}(y)\right|,
$$

and for all $y \in Y$ one has

$$
\left|\operatorname{Jac} F_{c}(y)\right| \leq 1+\alpha_{1} .
$$

Moreover, $\alpha_{1}(\varepsilon, \delta) \rightarrow 0$ as $\varepsilon$ and $\delta \rightarrow 0$.

Proof

Let

$$
\alpha=\max \left(\sqrt{\left(1+\frac{\delta}{n-1}\right)^{n}-1}, \sqrt{\varepsilon}\right) .
$$

Thus

$$
\left(1+\frac{\delta}{n-1}\right)^{n-1} \leq 1+\alpha^{2}
$$

and $\varepsilon \leq \alpha^{2}$. In particular, $\left|\operatorname{Jac} F_{c}(y)\right| \leq 1+\alpha^{2} \leq 1+\alpha$ for all $y \in Y$, if $\delta$ is small enough so that $\alpha$ is less than 1 . (We also assume that $\varepsilon$ is small.)

As $F_{c}$ has degree one, we have

$$
\operatorname{vol}_{g_{0}}(X)=\int_{Y} F_{c}^{*}\left(\mathrm{dv}_{\mathrm{g}_{0}}\right)=\int_{Y} \operatorname{Jac} F_{c}(y) \mathrm{dv}_{\mathrm{g}}(y) .
$$


Denote by $Y_{\alpha_{1}}$ the set of points $y \in Y$ such that

$$
\left|\operatorname{Jac} F_{c}(y)\right| \geq 1-\alpha .
$$

We have

$$
\begin{aligned}
\operatorname{vol}_{g_{0}}(X) & \leq \int_{Y}\left|\operatorname{Jac} F_{c}(y)\right| \operatorname{dv}_{\mathrm{g}}(y) \\
& =\int_{Y_{\alpha_{1}}}\left|\operatorname{Jac} F_{c}(y)\right| \operatorname{dv}_{\mathrm{g}}(y)+\int_{Y \backslash Y_{\alpha_{1}}}\left|\operatorname{Jac} F_{c}(y)\right| \operatorname{dv}_{\mathrm{g}}(y) \\
& \leq\left(1+\alpha^{2}\right) \operatorname{vol}_{g}\left(Y_{\alpha_{1}}\right)+(1-\alpha) \operatorname{vol}_{g}\left(Y \backslash Y_{\alpha_{1}}\right) \\
& =\operatorname{vol}_{g}(Y)+\alpha^{2} \operatorname{vol}_{g}\left(Y_{\alpha_{1}}\right)-\alpha \operatorname{vol}_{g}\left(Y \backslash Y_{\alpha_{1}}\right)
\end{aligned}
$$

Then, using assumption (4) and the inequality (5) on the volume, we get

$$
\begin{aligned}
\operatorname{vol}_{g}\left(Y \backslash Y_{\alpha_{1}}\right) & \leq \frac{\operatorname{vol}_{g}(Y)-\operatorname{vol}_{g_{0}}(X)}{\alpha}+\alpha \operatorname{vol}_{g}\left(Y_{\alpha_{1}}\right) \\
& \leq\left(\frac{\varepsilon}{\alpha}+\alpha\right) \operatorname{vol}_{g}(Y) \\
& \leq 2 \alpha \operatorname{vol}_{g}(Y) .
\end{aligned}
$$

Clearly, $1-2 \alpha \leq\left|\mathrm{Jac} F_{c}(y)\right|$ on $Y_{\alpha_{1}}$ and $\left|\mathrm{Jac} F_{c}(y)\right| \leq 1+2 \alpha$ on $Y$, which proves the lemma with $\alpha_{1}(\varepsilon, \delta)=2 \alpha$.

From this lemma, we deduce that $F_{c}$ is almost injective. Indeed, let $x \in X$; one defines $N\left(F_{c}, x\right) \in \mathbf{N} \cup\{\infty\}$ to be the number of preimages of $x$ by $F_{c}$. As $F_{c}$ has degree one, one has $N\left(F_{c}, x\right) \geq 1$ for all $x \in X$. We then define $X_{1}:=\{x \in$ $X, N\left(F_{c}, x\right)=1$ \}. Observe that $N\left(F_{c}, x\right) \geq 2$ on $X \backslash X_{1}$.

LEMMA 3.10

There exists $\alpha_{2}=\alpha_{2}(\varepsilon, \delta)>0$ such that

$$
\operatorname{vol}_{g_{0}}\left(X_{1}\right) \geq\left(1-\alpha_{2}\right) \operatorname{vol}_{g_{0}}(X)
$$

and

$$
\int_{X \backslash X_{1}} N\left(F_{c}, x\right) \mathrm{dv}_{\mathrm{g}_{0}}(x) \leq \alpha_{2}(\varepsilon, \delta) \operatorname{vol}_{g_{0}}(X) .
$$

Moreover, $\alpha_{2}(\varepsilon, \delta) \rightarrow 0$ as $\varepsilon$ and $\delta \rightarrow 0$.

In particular, there exists $\alpha^{\prime}>0$ such that $N\left(F_{c}, x\right)=1 \alpha^{\prime}$-a.e. on $X$. 
Proof

One defines

$$
\alpha_{2}(\varepsilon, \delta)=2\left(\left(1+\frac{\delta}{n-1}\right)^{n}(1+\varepsilon)-1\right) .
$$

From (15) and the area formula (see [16, Section 3.7]), we have

$$
\begin{aligned}
\left(\frac{c}{h\left(g_{0}\right)}\right)^{n} \operatorname{vol}_{g}(Y) \geq & \int_{Y}\left|\operatorname{Jac} F_{c}(y)\right| \mathrm{dv}_{\mathrm{g}}(y) \\
= & \int_{X} N\left(F_{c}, x\right) \operatorname{dv}_{\mathrm{g}_{0}}(x) \\
= & \int_{X_{1}} N\left(F_{c}, x\right) \mathrm{dv}_{\mathrm{g}_{0}}(x) \\
& +\int_{X \backslash X_{1}}\left(N\left(F_{c}, x\right)-1+1\right) \mathrm{dv}_{\mathrm{g}_{0}}(x) \\
= & \operatorname{vol}_{g_{0}}(X)+\int_{X \backslash X_{1}}\left(N\left(F_{c}, x\right)-1\right) \mathrm{dv}_{\mathrm{g}_{0}}(x)
\end{aligned}
$$

and

$$
\begin{aligned}
\operatorname{vol}_{g_{0}}\left(X \backslash X_{1}\right) & \leq \int_{X \backslash X_{1}}\left(N\left(F_{c}, x\right)-1\right) \mathrm{dv}_{\mathrm{g}_{0}}(x) \\
& \leq\left(\frac{c}{h\left(g_{0}\right)}\right)^{n} \operatorname{vol}_{g}(Y)-\operatorname{vol}_{g_{0}}(X) \\
& \leq\left(\left(\frac{c}{h\left(g_{0}\right)}\right)^{n}(1+\varepsilon)-1\right) \operatorname{vol}_{g_{0}}(X) \\
& \leq \frac{\alpha_{2}(\varepsilon, \delta)}{2} \operatorname{vol}_{g_{0}}(X) .
\end{aligned}
$$

Thus, since $N\left(F_{c}, x\right) \leq 2\left(N\left(F_{c}, x\right)-1\right)$ on $X \backslash X_{1}$, we get

$$
\operatorname{vol}_{g_{0}}\left(X \backslash X_{1}\right) \leq \int_{X \backslash X_{1}} N\left(F_{c}, x\right) \operatorname{dv}_{\mathrm{g}_{0}}(x) \leq \alpha_{2}(\varepsilon, \delta) \operatorname{vol}_{g_{0}}(X),
$$

and this proves the lemma.

The following lemma says that $d F_{c}(y)$ is almost isometric at points $y$ where Jac $F_{c}(y)$ is almost equal to 1 .

\section{LEMMA 3.11}

There exists $\alpha_{3}=\alpha_{3}(\varepsilon, \delta)>0$ such that the following holds. Let $Y_{\alpha_{1}}$ be the set of 
points where (18) holds, that is, $1-\alpha_{1}(\varepsilon, \delta) \leq\left|\operatorname{Jac} F_{c}(y)\right|$. Let $y$ be a point in $Y_{\alpha_{1}}$, and let $u \in T_{y} Y$; then

$$
\left(1-\alpha_{3}\right)\|u\|_{g} \leq\left\|d_{y} F_{c}(u)\right\|_{g_{0}} \leq\left(1+\alpha_{3}\right)\|u\|_{g} .
$$

Moreover, $\alpha_{3}(\varepsilon, \delta) \rightarrow 0$ as $\varepsilon, \delta \rightarrow 0$.

\section{Proof}

The inequality (15) implies that, for all $y \in Y$,

$$
\left\|H_{y}^{c}-\frac{1}{n} \operatorname{Id}\right\|^{2} \leq \frac{1}{A}\left(1-\frac{\left|\operatorname{Jac} F_{c}(y)\right|}{\left(1+\frac{\delta}{n-1}\right)^{n}}\right) .
$$

Let us define

$$
\beta_{1}=\beta_{1}(\varepsilon, \delta)=\frac{1}{A^{1 / 2}}\left(1-\frac{1-\alpha_{1}(\varepsilon, \delta)}{\left(1+\frac{\delta}{n-1}\right)^{n}}\right)^{1 / 2},
$$

where $\alpha_{1}(\varepsilon, \delta)$ is the constant from Lemma 3.9. Clearly, $\beta_{1}(\varepsilon, \delta) \rightarrow 0$ as $\varepsilon$ and $\delta \rightarrow 0$. Let $Y_{\alpha_{1}}$ be the set of points where (18) holds. On $Y_{\alpha_{1}}$, one has

$$
\left\|H_{y}^{c}-\frac{\mathrm{Id}}{n}\right\|^{2} \leq \beta_{1}{ }^{2} .
$$

Let $\left\{u_{i}\right\}_{i=1, \ldots, n}$ be an orthonormal basis of $T_{y} Y$, and let $v_{i}=d_{y} F\left(u_{i}\right)$. Writing Id $H_{y}^{c}=\frac{n-1}{n} \mathrm{Id}+\frac{1}{n} \mathrm{Id}-H_{y}^{c}$, one gets

$$
\begin{aligned}
\left|g_{0}\left(\left(\mathrm{Id}-H_{y}^{c}\right) d_{y} F_{c}\left(u_{i}\right), d_{y} F_{c}\left(u_{i}\right)\right)\right| \\
\geq\left|g_{0}\left(\left(\frac{n-1}{n} \mathrm{Id}\right) d_{y} F_{c}\left(u_{i}\right), d_{y} F_{c}\left(u_{i}\right)\right)\right| \\
\quad-\left|g_{0}\left(\left(\frac{1}{n} \mathrm{Id}-H_{y}^{c}\right) d_{y} F_{c}\left(u_{i}\right), d_{y} F_{c}\left(u_{i}\right)\right)\right| \\
\quad \geq \frac{n-1}{n}\left\|d_{y} F_{c}\left(u_{i}\right)\right\|_{g_{0}}^{2}-\left\|\frac{1}{n} \mathrm{Id}-H_{y}^{c}\right\| \cdot\left\|d_{y} F_{c}\left(u_{i}\right)\right\|_{g_{0}}^{2} \\
\geq\left(\frac{n-1}{n}-\beta_{1}\right)\left\|d_{y} F_{c}\left(u_{i}\right)\right\|_{g_{0}}^{2} .
\end{aligned}
$$

Writing $H_{y}^{c}=\frac{1}{n} \mathrm{Id}+H_{y}^{c}-\frac{1}{n} \mathrm{Id}$, one has

$$
\begin{aligned}
& g_{0}\left(H_{y}^{c} d_{y} F_{c}\left(u_{i}\right), d_{y} F_{c}\left(u_{i}\right)\right)^{1 / 2} \\
& \quad \leq g_{0}\left(\left(\frac{1}{n} \mathrm{Id}\right) d_{y} F_{c}\left(u_{i}\right), d_{y} F_{c}\left(u_{i}\right)\right)^{1 / 2}
\end{aligned}
$$




$$
\begin{aligned}
& +\left|g_{0}\left(\left(H_{y}^{c}-\frac{1}{n} \mathrm{Id}\right) d_{y} F_{c}\left(u_{i}\right), d_{y} F_{c}\left(u_{i}\right)\right)\right|^{1 / 2} \\
\leq & \left(\frac{1}{\sqrt{n}}+\beta_{1}^{1 / 2}\right)\left\|d_{y} F_{c}\left(u_{i}\right)\right\|_{g_{0}} .
\end{aligned}
$$

Taking the trace of the right-hand side of (13) and using the Cauchy-Schwarz inequality, one has

$$
\begin{aligned}
& \sum_{i=1}^{n} g_{0}\left(H_{y}^{c} d_{y} F_{c}\left(u_{i}\right), d_{y} F_{c}\left(u_{i}\right)\right)^{1 / 2} g\left(H_{y}^{\prime c}\left(u_{i}\right), u_{i}\right)^{1 / 2} \\
& \quad \leq\left(\frac{1}{\sqrt{n}}+\beta_{1}^{1 / 2}\right)\left(\sum_{i=1}^{n}\left\|d_{y} F_{c}\left(u_{i}\right)\right\|_{g_{0}}^{2}\right)^{1 / 2}\left(\sum_{i=1}^{n} g\left(H_{y}^{\prime c}\left(u_{i}\right), u_{i}\right)\right)^{1 / 2} \\
& \quad=\left(\frac{1}{\sqrt{n}}+\beta_{1}^{1 / 2}\right)\left(\sum_{i=1}^{n}\left\|d_{y} F_{c}\left(u_{i}\right)\right\|_{g_{0}}^{2}\right)^{1 / 2}
\end{aligned}
$$

By (13), the trace of (42) is not greater than the right-hand side of (46) multiplied by $c$, and hence

$$
\left(\frac{n-1}{n}-\beta_{1}\right) \sum_{i=1}^{n}\left\|d_{y} F_{c}\left(u_{i}\right)\right\|_{g_{0}}^{2} \leq c\left(\frac{1}{\sqrt{n}}+\beta_{1}^{1 / 2}\right)\left(\sum_{i=1}^{n}\left\|d_{y} F_{c}\left(u_{i}\right)\right\|_{g_{0}}^{2}\right)^{1 / 2},
$$

and

$$
\left(\sum_{i=1}^{n}\left\|d_{y} F_{c}\left(u_{i}\right)\right\|_{g_{0}}^{2}\right)^{1 / 2} \leq c \frac{\frac{1}{\sqrt{n}}+\beta_{1}^{1 / 2}}{\frac{n-1}{n}-\beta_{1}} \leq \sqrt{n}\left(1+\frac{\delta}{n-1}\right) \frac{1+\sqrt{n} \beta_{1}^{1 / 2}}{1-\frac{n}{n-1} \beta_{1}}
$$

Let us define

$$
\beta_{2}:=\beta_{2}(\varepsilon, \delta)=\left(1+\frac{\delta}{n-1}\right)^{2}\left(\frac{1+\sqrt{n} \beta_{1}^{1 / 2}}{1-\frac{n}{n-1} \beta_{1}}\right)^{2}-1
$$

Clearly, $\beta_{2}(\varepsilon, \delta) \rightarrow 0$ as $\varepsilon$ and $\delta \rightarrow 0$. One has

$$
\sum_{i=1}^{n}\left\|d_{y} F_{c}\left(u_{i}\right)\right\|_{g_{0}}^{2} \leq n\left(1+\beta_{2}\right)
$$

Let $L$ be the endomorphism of $T_{y} Y$ defined by $L=\left(d_{y} F_{c}\right)^{*} \circ d_{y} F_{c}$. We have

$$
\operatorname{trace}(L)=\sum_{i=1}^{n} g\left(L\left(u_{i}\right), u_{i}\right)=\sum_{i=1}^{n} g\left(d_{y} F_{c}\left(u_{i}\right), d_{y} F_{c}\left(u_{i}\right)\right) \leq n\left(1+\beta_{2}\right)
$$


On the other hand,

$$
|1-\alpha|^{2} \leq\left|\operatorname{Jac} F_{c}(y)\right|^{2}=\operatorname{det}(L) \leq\left(\frac{\operatorname{trace}(L)}{n}\right)^{n} \leq\left(1+\beta_{2}\right)^{n},
$$

which shows that there is almost equality in the arithmetic-geometric inequality. We then get that there exists some $\alpha_{3}(\varepsilon, \delta)>0$, with $\alpha_{3}(\varepsilon, \delta) \rightarrow 0$ as $\varepsilon, \delta \rightarrow 0$, such that

$$
\|L-\mathrm{Id}\| \leq \alpha_{3}(\varepsilon, \delta) .
$$

Thus for any $y \in Y_{\alpha_{1}}$ and $u \in T_{y} Y$,

$$
\left(1-\alpha_{3}\right)\|u\| \leq\left\|d_{y} F_{c}(u)\right\|_{g_{0}} \leq\left(1+\alpha_{3}\right)\|u\|,
$$

and $d_{y} F_{c}$ is almost isometric.

We now prove that given a fixed radius $R>0$, the natural maps $F_{c}$ have uniformly bounded differential $d F_{c}$ on $B\left(y_{g}, R\right)$ if the parameters $\varepsilon, \delta$ are sufficiently small. Recall that the point $y_{g}$ has been chosen such that (8) holds; namely, $\operatorname{vol}_{g}\left(B\left(y_{g}, 1\right)\right) \geq v_{n}$.

\section{LEMMA 3.12}

Let $R>0$. Then there exist $\varepsilon(R)>0$ and $\delta(R)>0$ such that for any $0<\varepsilon<\varepsilon(R)$ and $0<\delta<\delta(R)$, and for any $y \in B\left(y_{g}, R\right)$,

$$
\left\|d_{y} F_{c}\right\| \leq 2 \sqrt{n} .
$$

\section{Proof}

We first prove that for all $y \in Y,\left\|d_{y} F_{c}\right\|$ is bounded from above by $\lambda_{n}^{c}(y)$, the maximal eigenvalue of $H_{y}^{c}$ (see Definition 3.4). Recall that all eigenvalues of $H_{y}^{\prime c}$ are less than one and that $0<\lambda_{n}^{c}<1$. Let $u$ be a unit vector in $T_{y} \tilde{Y}$, and let $v=d_{y} F_{c}(u)$. Equation (13) gives

$$
\left(1-\lambda_{n}^{c}(y)\right)\left|g_{0}\left(d_{y} F_{c}(u), d_{y} F_{c}(u)\right)\right| \leq c \lambda_{n}^{c}(y)^{1 / 2} g_{0}\left(d_{y} F_{c}(u), d_{y} F_{c}(u)\right)^{1 / 2} .
$$

Hence

$$
\left\|d_{y} F_{c}(u)\right\|_{g_{0}} \leq \frac{c \sqrt{\lambda_{n}^{c}(y)}}{1-\lambda_{n}^{c}(y)} .
$$

We thus have to show that $\lambda_{n}^{c}(y)$ is not close to 1 . More precisely, let $\beta>0$ such that $\frac{1}{n}+\beta<1$; one then defines

$$
\gamma(\delta, \beta):=\left(\frac{n-1+\delta}{n-1-n \beta}\right) \sqrt{1+n \beta}-1>0 .
$$


Clearly, $\gamma(\beta, \delta) \rightarrow 0$ as $\delta, \beta \rightarrow 0$. One can check that if $\lambda_{n}^{c}(y) \leq \frac{1}{n}+\beta$, then $\left\|d_{y} F_{c}(u)\right\|_{g_{0}} \leq \sqrt{n}(1+\gamma)$. For our purpose, we may suppose that $\gamma \leq 1$. Now let $\delta_{n}>0$ and $\beta_{n}>0$ be such that if $0<\delta \leq 10 \delta_{n}$ and $0<\beta \leq 10 \beta_{n}$, then $\gamma(\delta, \beta) \leq 1$. Moreover, we define $\varepsilon_{n}>0$ such that if $0<\varepsilon<\varepsilon_{n}$ and $0<\delta \leq 10 \delta_{n}$, then with the notation of (38) and Lemma 3.9, $\beta_{1}(\varepsilon, \delta) \leq \beta_{n}$. In what follows, we suppose $\varepsilon$ and $\delta$ to be sufficiently small.

By (39) we have that $\left|\lambda_{n}^{c}(y)-\frac{1}{n}\right| \leq \beta_{1}(\varepsilon, \delta)$ on $Y_{\alpha_{1}}$. Recall that $Y_{\alpha_{1}}$ has a large relative volume in $Y$. The idea is first to estimate $\lambda_{n}^{c}$ on a neighborhood of $Y_{\alpha_{1}}$ and then to show that this neighborhood contains $B\left(y_{g}, R\right)$ if the parameters $\varepsilon$ and $\delta$ are sufficiently small relative to $R$.

For this purpose we need to estimate the variation of $\lambda_{n}^{c}$. Recall that $H_{y}^{c}$ is defined by

$$
g_{0}\left(H_{y}^{c}(u), v\right)=\int_{\partial \tilde{X}} d B_{\left(F_{c}(y), \theta\right)}(u) d B_{\left(F_{c}(y), \theta\right)}(v) d \sigma_{y}^{c}(\theta) .
$$

Let $U, V$ be parallel vector fields near $F_{c}(y)$ extending unit tangent vectors at $F_{c}(y)$, $u$, and $v$. We compute the derivative of $g_{0}\left(H_{y}^{c}(U), V\right)$ in a direction $w \in T_{y} Y$ :

$$
\begin{aligned}
w \cdot g_{0}\left(H_{y}^{c}(U), V\right)= & \int_{\partial \tilde{X}} D d B_{\left(F_{c}(y), \theta\right)}\left(d_{y} F(w), U\right) d B_{\left(F_{c}(y), \theta\right)}(V) d \sigma_{y}^{c}(\theta) \\
& +\int_{\partial \tilde{X}} d B_{\left(F_{c}(y), \theta\right)}(U) D d B_{\left(F_{c}(y), \theta\right)}\left(d_{y} F(w), V\right) d \sigma_{y}^{c}(\theta) \\
& +\int_{\partial \tilde{X}} d B_{\left(F_{c}(y), \theta\right)}(U) d B_{\left(F_{c}(y), \theta\right)}(V) w \cdot d \sigma_{y}^{c}(\theta) .
\end{aligned}
$$

The Buseman functions of the hyperbolic space satisfies $\|D d B\| \leq 1$ and $\|d B\| \leq 1$, and thus

$$
\left|w \cdot g_{0}\left(H_{y}^{c}(U), V\right)\right| \leq 2\left\|d_{y} F_{c}(w)\right\|_{g_{0}}+\left|\int_{\partial \tilde{X}} w \cdot d \sigma_{y}^{c}(\theta)\right| .
$$

Recall that

$$
d \sigma_{y}^{c}(\theta)=\frac{d \mu_{y}^{c}(\theta)}{\mu_{y}^{c}(\partial \tilde{X})}=\frac{\int_{\tilde{Y}} p(\tilde{f}(z), \theta) e^{-c \rho(y, z)} \mathrm{dv}_{\tilde{\mathrm{g}}}(z)}{\int_{\tilde{Y}} e^{-c \rho(y, z)} \mathrm{dv}_{\tilde{\mathrm{g}}}(z)} d \theta .
$$

Differentiating this formula yields

$$
\begin{aligned}
w \cdot d \sigma_{y}^{c}(\theta)= & \frac{\int_{\tilde{Y}} p(\tilde{f}(z), \theta)\left(-c \cdot d \rho_{(y, z)}(w)\right) e^{-c \rho(y, z)} \mathrm{dv}_{\tilde{\mathrm{g}}}(z)}{\mu_{y}^{c}(\partial \tilde{X})} d \theta \\
& -\frac{d \mu_{y}^{c}(\theta)}{\mu_{y}^{c}(\partial \tilde{X})^{2}} \cdot \int_{\tilde{Y}}\left(-c \cdot d \rho_{(y, z)}(w)\right) e^{-c \rho(y, z)} \mathrm{dv}_{\tilde{\mathrm{g}}}(z) .
\end{aligned}
$$


Since $\left|d \rho_{(y, z)}(w)\right| \leq\|w\|_{g}$, we have

$$
\left|\int_{\partial \tilde{X}} w \cdot d \sigma_{y}^{c}(\theta)\right| \leq \int_{\partial \tilde{X}} 2 c\|w\|_{g} d \sigma_{y}^{c}(\theta)=2 c\|w\|_{g},
$$

which gives $\left|w \cdot g_{0}\left(H_{y}^{c}(U), V\right)\right| \leq 2\left\|d_{y} F_{c}(w)\right\|_{g_{0}}+2 c\|w\|_{g}$. If $w$ is a unit vector, then (51) yields

$$
\left|w \cdot g_{0}\left(H_{y}^{c}(U), V\right)\right| \leq 2 c\left(\frac{\sqrt{\lambda_{n}^{c}(y)}}{1-\lambda_{n}^{c}(y)}+1\right) .
$$

Let us now consider small constants $\eta>\beta>0$ and define

$$
r(\delta, \beta, \eta):=\frac{\eta-\beta}{2(n-1+\delta)\left(\frac{\sqrt{\frac{1}{n}+\eta}}{1-\left(\frac{1}{n}+\eta\right)}+1\right)}>0 .
$$

Our goal is to prove that

$$
\inf \left\{d\left(y_{0}, y_{1}\right) \mid y_{0}, y_{1} \in Y, \lambda_{n}^{c}\left(y_{0}\right) \leq \frac{1}{n}+\beta, \lambda_{n}^{c}\left(y_{1}\right) \geq \frac{1}{n}+\eta\right\} \geq r(\delta, \beta, \eta) .
$$

Let $y_{0} \in Y$ so that $\lambda_{n}^{c}\left(y_{0}\right) \leq \frac{1}{n}+\beta$. Assume that there exists $y \in Y$ such that $\lambda_{n}^{c}(y) \geq$ $\frac{1}{n}+\eta$. One defines

$$
r:=\inf \left\{d\left(y_{0}, y\right) \mid y \in Y, \lambda_{n}^{c}(y) \geq \frac{1}{n}+\eta\right\} .
$$

By continuity, there exists $y_{1} \in Y$ such that $\lambda_{n}^{c}\left(y_{1}\right)=\frac{1}{n}+\eta$ and $d\left(y_{0}, y_{1}\right)=r$.

Let $\gamma:[0, r] \longrightarrow Y$ be a minimizing geodesic from $y_{0}$ to $y_{1}$. We easily see that $\lambda_{n}^{c}(\gamma(t))<\frac{1}{n}+\eta$ for any $0 \leq t<r$. Let $U(t)$ be a parallel vector field in $X$ along $F_{c}(\gamma)$ such that $U(r)$ is a unit eigenvector of $H_{y_{1}}^{c}$. Then, using (55) with $\dot{\gamma} \cdot g_{0}\left(H_{\gamma(t)}^{c} U(t), U(t)\right)=\frac{d}{d t} g_{0}\left(H_{\gamma(t)}^{c} U(t), U(t)\right)$, one has

$$
\begin{aligned}
\left|\lambda_{n}^{c}\left(y_{1}\right)-\lambda_{n}^{c}\left(y_{0}\right)\right| & \leq\left|g_{0}\left(H_{\gamma(r)}^{c} U(r), U(r)\right)-g_{0}\left(H_{\gamma(0)}^{c} U(0), U(0)\right)\right| \\
& =\left|\int_{0}^{r} \frac{d}{d t} g_{0}\left(H_{\gamma(t)}^{c} U(t), U(t)\right) d t\right| \\
& \leq 2 c \int_{0}^{r}\left(\frac{\sqrt{\lambda_{n}^{c}(\gamma(t))}}{1-\lambda_{n}^{c}(\gamma(t))}+1\right) d t \\
& \leq 2 c r\left(\frac{\sqrt{\frac{1}{n}+\eta}}{1-\left(\frac{1}{n}+\eta\right)}+1\right) .
\end{aligned}
$$


As a consequence,

$$
r \geq \frac{\eta-\beta}{2(n-1+\delta)\left(\frac{\sqrt{\frac{1}{n}+\eta}}{1-\left(\frac{1}{n}+\eta\right)}+1\right)}=r(\delta, \beta, \eta) .
$$

We now set $\eta=2 \beta_{n}$ so that $\gamma(\delta, \eta) \leq 1$ for any $\delta \leq \delta_{n}$. One then defines $r_{n}:=$ $r\left(\delta_{n}, \beta_{n}, 2 \beta_{n}\right)$. Let us recall that for $\varepsilon \leq \varepsilon_{n}$ and $\delta \leq \delta_{n}$, we have $\beta_{1}(\varepsilon, \delta) \leq \beta_{n}$. On $Y_{\alpha_{1}}$, one has $\lambda_{n}^{c}(y) \leq \frac{1}{n}+\beta_{1}(\varepsilon, \delta) \leq \frac{1}{n}+\beta_{n}$. Hence, if $\lambda_{n}^{c}\left(y_{1}\right) \geq \frac{1}{n}+2 \beta_{n}$, one has

$$
d\left(y_{1}, Y_{\alpha_{1}}\right) \geq r\left(\delta, \beta_{1}(\varepsilon, \delta), 2 \beta_{n}\right) \geq r\left(\delta_{n}, \beta_{n}, 2 \beta_{n}\right)=r_{n}
$$

We thus have proved that in the $r_{n}$-neighborhood of $Y_{\alpha_{1}}$, one has $\lambda_{n}^{c}(y) \leq \frac{1}{n}+2 \beta_{n}$. This implies that

$$
\left\|d_{y} F_{c}\right\| \leq\left(1+\gamma\left(\delta, 2 \beta_{n}\right)\right) \sqrt{n} \leq 2 \sqrt{n}
$$

Let us denote by $V_{r_{n}}\left(Y_{\alpha_{1}}\right)$ the $r_{n}$-neighborhood of $Y_{\alpha_{1}}$. It remains to show that $B\left(y_{g}, R\right) \subset V_{r_{n}}\left(Y_{\alpha_{1}}\right)$, if $\varepsilon \leq \varepsilon(R)$ and $\delta \leq \delta(R)$. Let us recall that

$$
\frac{\operatorname{vol}_{g}\left(Y_{\alpha_{1}}\right)}{\operatorname{vol}_{g}(Y)} \geq 1-\alpha_{1}
$$

and hence

$$
\operatorname{vol}_{g}\left(Y \backslash Y_{\alpha_{1}}\right) \leq \alpha_{1} \operatorname{vol}_{g}(Y) \leq \alpha_{1}(1+\varepsilon) \operatorname{vol}_{g_{0}}(X):=v(\varepsilon, \delta)
$$

Clearly, $v(\varepsilon, \delta) \rightarrow 0$ when $\varepsilon, \delta \rightarrow 0$. On the other hand, by (11) for any $y \in B\left(y_{g}, R\right)$, we have

$$
\operatorname{vol}_{g}\left(B_{g}\left(y, r_{0}\right)\right) \geq v_{n} \frac{\operatorname{vol}_{\mathbf{H}^{n}}\left(\mathrm{~B}_{\mathbf{H}^{n}}\left(r_{0}\right)\right)}{\operatorname{vol}_{\mathbf{H}^{n}}\left(\mathrm{~B}_{\mathbf{H}^{n}}\left(1+R+r_{0}\right)\right)}:=v_{0}(R)>0 .
$$

If $v_{0}(R)>v(\varepsilon, \delta)$, then for any $y \in B\left(y_{g}, R\right)$ one has $B_{g}\left(y, r_{n}\right) \not \subset Y \backslash Y_{\alpha_{1}}$, which means that $B_{g}\left(y, r_{n}\right)$ intersects $Y_{\alpha_{1}}$. This shows that $d\left(y, Y_{\alpha_{1}}\right)<r_{n}$ and $y \in V_{r_{n}}\left(Y_{\alpha_{1}}\right)$.

The lemma is proved if we define $\varepsilon=\varepsilon(R)>0$ and $\delta=\delta(R)>0$ to be sufficiently small constants such that $v(\varepsilon, \delta)<v_{0}(R)$.

We now prove that $F_{c}$ is almost 1-Lipschitz.

\section{LEMMA 3.13}

For any fixed $R>0$, there exists $\varepsilon_{2}(R)>0$ and $\delta_{2}(R)>0$ such that for every $0<\varepsilon<$ $\varepsilon_{2}(R)$ and $0<\delta<\delta_{2}(R)$, there exists $\kappa=\kappa(\varepsilon, \delta, R)>0$ such that on $B_{g}\left(y_{g}, R\right)$,

$$
d_{g_{0}}\left(F_{c}\left(y_{1}\right), F_{c}\left(y_{2}\right)\right) \leq(1+\kappa) d_{g}\left(y_{1}, y_{2}\right)+\kappa .
$$


Moreover, $\kappa(\varepsilon, \delta, R) \rightarrow 0$ as $\varepsilon, \delta \rightarrow 0$.

\section{Proof}

The idea goes as follows. We have proved that $d_{y} F_{c}$ is almost isometric on $Y_{\alpha_{1}}$. On the other hand, $\left\|d_{y} F_{c}\right\|$ is uniformly bounded in $B\left(y_{g}, R\right)$ if the parameters $\varepsilon$ and $\delta$ are chosen sufficiently small. To prove the lemma one computes the lengths of $F_{c}(\gamma)$ where $\gamma$ is a minimizing geodesic in $B\left(y_{g}, R\right)$ whose intersection with $Y_{\alpha_{1}}$ is large. Existence of such geodesics follows from an integral geometry lemma due to T. Colding.

Fix some $R>0$. We define the following constants.

If $d>0$,

$$
c_{1}(n, d):=\sup _{0<s / 2<r<s<d} \frac{\operatorname{vol}_{\mathbf{H}^{n}}\left(\partial B_{\mathbf{H}^{n}}(s)\right)}{\operatorname{vol}_{\mathbf{H}^{n}}\left(\partial B_{\mathbf{H}^{n}}(r)\right)} .
$$

If $\tau>0, R>0$,

$$
c_{2}(n, \tau, R):=c_{1}(n, 2 R)\left(2 \tau \operatorname{vol}_{\mathbf{H}^{n}}\left(\mathrm{~B}_{\mathbf{H}^{n}}(\tau)\right)\right) .
$$

If $\varepsilon>0, \delta>0$,

$$
\theta(\varepsilon, \delta):=2 \alpha_{3}^{2}(\varepsilon, \delta) \operatorname{vol}_{g_{0}}(X)+2(4 n+1) \alpha_{1}(\varepsilon, \delta) \operatorname{vol}_{g_{0}}(X) .
$$

Clearly, $\theta(\varepsilon, \delta) \rightarrow 0$ as $\varepsilon, \delta \rightarrow 0$.

Let $\tau(\varepsilon, \delta, R)>0$ be the function implicitly defined by

$$
\operatorname{vol}_{\mathbf{H}^{n}}(\tau) \tau:=\theta(\varepsilon, \delta) \frac{2 c_{1}(n, 2 R) \operatorname{vol}_{\mathbf{H}^{n}}(1+R+1)^{2}}{v_{n}^{2}} .
$$

Again, we easily see that, for fixed $R, \tau(\varepsilon, \delta, R) \rightarrow 0$ as $\varepsilon, \delta \rightarrow 0$. We also choose $\varepsilon_{2}(R)>0$ and $\delta_{2}(R)>0$ such that $\varepsilon_{2}(R) \leq \varepsilon(2 R), \delta_{2}(R)<\delta(2 R)$ and such that, if $0<\varepsilon \leq \varepsilon_{2}(R)$ and $0<\delta<\delta_{2}(R)$, then $\tau(\varepsilon, \delta, R) \ll 1$.

Finally, one defines $\kappa(\varepsilon, \delta, R):=\max (2 \sqrt{n} \sqrt{\tau}, 8 \sqrt{\tau})$. From the remarks above we can choose $\varepsilon_{2}(R)$ and $\delta_{2}(R)$ so that $\kappa(\varepsilon, \delta, R)<1 / R$ (for $0<\varepsilon \leq \varepsilon_{2}(R), 0<\delta<$ $\delta_{2}(R)$ and $R$ big).

There are two cases.

Case (i). Let $y_{1}, y_{2}$ in $B_{g}\left(y_{g}, R\right)$ be such that $d\left(y_{1}, y_{2}\right) \leq \sqrt{\tau}$. Using (49), if $0<\varepsilon<\varepsilon(2 R), 0<\delta<\delta(2 R)$ one has

$$
d\left(F_{c}\left(y_{1}\right), F_{c}\left(y_{2}\right)\right) \leq 2 \sqrt{n} \sqrt{\tau} \leq \kappa .
$$

Case (ii). Let $y_{1}, y_{2}$ in $B_{g}\left(y_{g}, R\right)$ be such that $d\left(y_{1}, y_{2}\right) \geq \sqrt{\tau}$. We will use the following theorem, due to J. Cheeger and T. Colding (see [7, Theorem 2.11]), which we describe now in a particular case. We keep the notations of [7]. 
Let us define $A_{1}=B_{g}\left(y_{1}, \tau\right), A_{2}=B_{g}\left(y_{2}, \tau\right)$ and $W=B_{g}\left(y_{g}, 2 R\right)$ where $y_{1}$ and $y_{2}$ are points as above sitting on a complete Riemannian manifold $(Y, g)$ with $\operatorname{Ric}_{g} \geq-(n-1) g$. For any $z_{1} \in A_{1}$ and any unit vector $v_{1} \in T_{z_{1}} Y$, the set $I\left(z_{1}, v_{1}\right)$ defined by

$$
I\left(z_{1}, v_{1}\right)=\left\{t \mid \gamma(t) \in A_{2}, \gamma_{\mid[0, t]} \text { is minimal, } \gamma^{\prime}(0)=v_{1}\right\}
$$

has a measure $\left|I\left(z_{1}, v_{1}\right)\right|$ bounded above by $2 \tau$. Thus

$$
D\left(A_{1}, A_{2}\right):=\sup _{z_{1}, v_{1}}\left|I\left(z_{1}, v_{1}\right)\right| \leq 2 \tau,
$$

and, similarly, $D\left(A_{2}, A_{1}\right) \leq 2 \tau$. For any $z_{1} \in A_{1}$ and $z_{2} \in A_{2}$, let $\gamma_{z_{1} z_{2}}$ be a minimizing geodesic from $z_{1}$ to $z_{2}$. Clearly, $\gamma \subset B\left(y_{g}, 2 R\right)$. Then, by [7, Theorem 2.11], we have for any nonnegative integrable function $e$ defined on $Y$,

$$
\begin{aligned}
\int_{A_{1} \times A_{2}} & \int_{0}^{d\left(z_{1}, z_{2}\right)} e\left(\gamma_{z_{1}, z_{2}}\right)(s) d s \\
\leq & c_{1}(n, 2 R)\left(D\left(A_{1}, A_{2}\right) \operatorname{vol}\left(A_{1}\right)+D\left(A_{2}, A_{1}\right) \operatorname{vol}\left(A_{2}\right)\right) \\
& \times \int_{W} e(y) \mathrm{dv}_{\mathrm{g}}(y) .
\end{aligned}
$$

By Bishop's theorem, for $i=1,2$ we have

$$
\operatorname{vol}_{g}\left(A_{i}\right) \leq \operatorname{vol}_{\mathbf{H}^{n}}\left(\mathrm{~B}_{\mathbf{H}^{n}}(\tau)\right),
$$

and thus

$$
c_{1}(n, 2 R)\left(D\left(A_{1}, A_{2}\right) \operatorname{vol}\left(A_{1}\right)+D\left(A_{2}, A_{1}\right) \operatorname{vol}\left(A_{2}\right)\right) \leq c_{2}(n, \tau, R) .
$$

Therefore, applying (63) to the function

$$
e(y)=\sup _{u \in U_{y} Y}\left(\left\|d_{y} F_{c}(u)\right\|-\|u\|\right)^{2}
$$

and using (37) on $W \cap Y_{\alpha_{1}}$ and (49) on $W \backslash Y_{\alpha_{1}}$, we get

$$
\begin{aligned}
& \int_{A_{1} \times A_{2}} \int_{0}^{d\left(z_{1}, z_{2}\right)} e\left(\gamma_{z_{1}, z_{2}}\right)(s) d s \\
& \quad \leq c_{2}(n, \tau, R)\left(\int_{W \cap Y_{\alpha_{1}}} e(y) \mathrm{dv}_{\mathrm{g}}(y)+\int_{W \backslash Y_{\alpha_{1}}} e(y) \mathrm{dv}_{\mathrm{g}}(y)\right) \\
& \quad \leq c_{2}(n, \tau, R)\left(\alpha_{3}^{2} \cdot \operatorname{vol}_{g}(Y)+(4 n+1) \operatorname{vol}_{g}\left(Y \backslash Y_{\alpha_{1}}\right)\right) \\
& \quad \leq c_{2}(n, \tau, R) \theta(\varepsilon, \delta) .
\end{aligned}
$$


Now, if we denote by $\gamma:=\gamma_{z_{1} z_{2}}$, then we have

$$
\begin{aligned}
\left|\ell\left(F_{c} \circ \gamma\right)-\ell(\gamma)\right| & =\left|\int_{0}^{d\left(z_{1}, z_{2}\right)}\left\|d_{\gamma(s)} F_{c}(\dot{\gamma})\right\|-\|\dot{\gamma}\| d s\right| \\
& \leq \int_{0}^{d\left(z_{1}, z_{2}\right)} \sup _{u \in T_{y} Y}\left|\left\|d_{\gamma(s)} F_{c}(u)\right\|-\|u\|\right| d s .
\end{aligned}
$$

Using Cauchy-Schwarz inequality we have

$$
\begin{aligned}
\frac{\left|\ell\left(F_{c} \circ \gamma\right)-\ell(\gamma)\right|^{2}}{d\left(z_{1}, z_{2}\right)} & \leq \frac{\left(\int_{0}^{d\left(z_{1}, z_{2}\right)} \sup _{u}\left|\left\|d_{\gamma(s)} F_{c}(u)\right\|-\|u\|\right| d s\right)^{2}}{d\left(z_{1}, z_{2}\right)} \\
& \leq \int_{0}^{d\left(z_{1}, z_{2}\right)} e(\gamma(s)) d s .
\end{aligned}
$$

Integrating on $A_{1} \times A_{2}$, we deduce from (64) that

$$
\int_{A_{1} \times A_{2}} \frac{\left|\ell\left(F_{c} \circ \gamma_{z_{1} z_{2}}\right)-\ell\left(\gamma_{z_{1} z_{2}}\right)\right|^{2}}{d\left(z_{1}, z_{2}\right)} \mathrm{dv}_{\mathrm{g}}\left(z_{1}\right) \mathrm{dv}_{\mathrm{g}}\left(z_{2}\right) \leq c_{2}(n, \tau, R) \theta(\varepsilon, \delta) .
$$

By (11), for $i=1,2$ one has

$$
\operatorname{vol}_{g}\left(A_{i}\right) \geq v_{n} \frac{\operatorname{vol}_{\mathbf{H}^{n}}\left(\mathrm{~B}_{\mathbf{H}^{n}}(\tau)\right)}{\operatorname{vol}_{\mathbf{H}^{n}}\left(\mathrm{~B}_{\mathbf{H}^{n}}(1+R+\tau)\right)}:=v_{0}(\tau, R)>0 .
$$

From the obvious inequality

$$
c_{2}(n, \tau, R) \theta(\varepsilon, \delta) \leq \frac{1}{v_{0}(\tau, R)^{2}} \int_{A_{1} \times A_{2}} c_{2}(n, \tau, R) \theta(\varepsilon, \delta) \operatorname{dv}_{\mathrm{g}}\left(z_{1}\right) \operatorname{dv}_{\mathrm{g}}\left(z_{2}\right),
$$

we get

$$
\int_{A_{1} \times A_{2}} \frac{\left|\ell\left(F_{c} \circ \gamma_{z_{1} z_{2}}\right)-\ell\left(\gamma_{z_{1} z_{2}}\right)\right|^{2}}{d\left(z_{1}, z_{2}\right)} \leq \int_{A_{1} \times A_{2}} \frac{c_{2}(n, \tau, R) \theta(\varepsilon, \delta)}{v_{0}(\tau, R)^{2}} .
$$

As a consequence there exist $z_{1} \in A_{1}$ and $z_{2} \in A_{2}$ such that

$$
\left|\ell\left(F_{c} \circ \gamma_{z_{1} z_{2}}\right)-\ell\left(\gamma_{z_{1} z_{2}}\right)\right|^{2} \leq d\left(z_{1}, z_{2}\right) \frac{c_{2}(n, \tau, R) \theta(\varepsilon, \delta)}{v_{0}(\tau, R)^{2}} .
$$

On the other hand, one can check that by definition of $\tau$,

$$
\frac{c_{2}(n, \tau, R) \theta(\varepsilon, \delta)}{v_{0}(\tau, R)^{2}}=\theta(\varepsilon, \delta) \frac{2 c_{1}(n, 2 R) \operatorname{vol}_{\mathbf{H}^{n}}(1+R+1)^{2}}{v_{n}^{2} \operatorname{vol}_{\mathbf{H}^{n}}(\tau)} \tau=\tau^{2} .
$$

This yields

$$
\left|\ell\left(F_{c} \circ \gamma_{z_{1} z_{2}}\right)-\ell\left(\gamma_{z_{1} z_{2}}\right)\right|^{2} \leq d\left(z_{1}, z_{2}\right) \tau^{2},
$$


and

$$
d\left(F_{c}\left(z_{1}\right), F_{c}\left(z_{2}\right)\right) \leq \ell\left(F_{c} \circ \gamma_{z_{1} z_{2}}\right) \leq d\left(z_{1}, z_{2}\right)+\tau \sqrt{d\left(z_{1}, z_{2}\right)} .
$$

Since $d\left(y_{i}, z_{i}\right)<\tau$ and $d\left(y_{1}, y_{2}\right) \geq \sqrt{\tau}$, we have

$$
d\left(z_{1}, z_{2}\right) \leq d\left(y_{1}, y_{2}\right)+2 \tau \leq d\left(y_{1}, y_{2}\right)(1+2 \sqrt{\tau}) .
$$

With our choice of $\tau$ very small compared to 1 , we also have

$$
d\left(z_{1}, z_{2}\right) \geq d\left(y_{1}, y_{2}\right)-2 \tau \geq \frac{\sqrt{\tau}}{2} .
$$

We then have

$$
\begin{aligned}
d\left(F_{c}\right. & \left.\left(y_{1}\right), F_{c}\left(y_{2}\right)\right) \\
& \leq d\left(F_{c}\left(y_{1}\right), F_{c}\left(z_{1}\right)\right)+d\left(F_{c}\left(z_{1}\right), F_{c}\left(z_{2}\right)\right)+d\left(F_{c}\left(z_{2}\right), F_{c}\left(y_{2}\right)\right) \\
& \leq 2 \sqrt{n} \tau+d\left(z_{1}, z_{2}\right)+\tau\left(d\left(z_{1}, z_{2}\right)\right)^{1 / 2}+2 \sqrt{n} \tau \\
& \leq 4 \sqrt{n} \tau+d\left(y_{1}, y_{2}\right) \frac{d\left(z_{1}, z_{2}\right)}{d\left(y_{1}, y_{2}\right)}\left(1+\tau\left(d\left(z_{1}, z_{2}\right)\right)^{-1 / 2}\right) \\
& \leq 4 \sqrt{n} \tau+d\left(y_{1}, y_{2}\right)(1+2 \sqrt{\tau})\left(1+\sqrt{2} \tau^{3 / 4}\right) \\
& \leq 4 \sqrt{n} \tau+d\left(y_{1}, y_{2}\right)(1+8 \sqrt{\tau}) .
\end{aligned}
$$

We finally get

$$
d\left(F_{c}\left(y_{1}\right), F_{c}\left(y_{2}\right)\right) \leq \kappa+(1+\kappa) d\left(y_{1}, y_{2}\right),
$$

in case (ii).

\section{A limit map on the limit space}

In this section, we consider a sequence $\left(Y_{k}, g_{k}\right)_{k \in \mathbf{N}}$ of closed Riemannian $n$-manifolds satisfying the curvature bound (3) and the assumption that there exist an closed hyperbolic $n$-manifold $\left(X, g_{0}\right)$, degree-one maps $f_{k}: Y_{k} \rightarrow X$, and a sequence $\varepsilon_{k} \rightarrow$ 0 such that

$$
\operatorname{vol}_{g_{k}}\left(Y_{k}\right) \rightarrow \operatorname{vol}_{g_{0}}(X),
$$

as $k$ goes to $+\infty$. From (8), for every $k \in \mathbf{N}$, there exists $y_{g_{k}} \in Y_{k}$ satisfying the local volume estimate; that is, $\operatorname{vol}\left(B_{g_{k}}\left(y_{g_{k}}, 1\right)\right) \geq v_{n}>0$. For the sake of simplicity we shall use the notation $y_{k}$ instead of $y_{g_{k}}$.

Below, we prove that $\left(Y_{k}, g_{k}, y_{k}\right)$ subconverges in the pointed Gromov-Haudorff topology to a limit metric space $\left(Y_{\infty}, d_{\infty}, z_{\infty}\right)$. Moreover, there exists a sequence of 
natural maps $F_{c_{k}}:\left(Y_{k}, g_{k}\right) \rightarrow\left(X, g_{0}\right)$, with suitably chosen parameters $c_{k}$, which subconverges to a "natural map" $F: Y_{\infty} \longrightarrow X$.

Let us recall the definition of the Gromov-Hausdorff topology. For two subsets $A, B$ of a metric space $Z$ the Hausdorff distance between $A$ and $B$ is

$$
\mathrm{d}_{\mathscr{H}}^{Z}(A, B):=\inf \left\{\varepsilon>0 \mid B \subset V_{\varepsilon}(A) \text { and } A \subset V_{\varepsilon}(B)\right\} \in \mathbf{R} \cup\{\infty\} .
$$

It is a distance on compact subsets of $Z$ (see [11]).

Definition 4.1 (see [14])

Let $X_{1}, X_{2}$ be two metric spaces. Then the Gromov-Hausdorff distance $\mathrm{d} \mathscr{\mathscr { H }}\left(X_{1}\right.$, $\left.X_{2}\right) \in \mathbf{R} \cup \infty$ is the infimum of the numbers

$$
\mathrm{d}_{\mathscr{H}}^{Z}\left(f_{1}\left(X_{1}\right), f_{2}\left(X_{2}\right)\right)
$$

for all metric spaces $Z$ and all isometric embeddings $f_{i}: X_{i} \rightarrow Z$.

It is a distance on the space of isometry classes of compact metric spaces. One says that a sequence $\left(X_{i}\right)_{i \in \mathbf{N}}$ of metric spaces converges in the Gromov-Hausdorff topology to a metric space $X_{\infty}$ if $\mathrm{d} \mathscr{\mathscr { H }}\left(X_{i}, X_{\infty}\right) \rightarrow 0$ as $i \rightarrow \infty$. Let $x_{i} \in X_{i}$ and $x_{\infty} \in X_{\infty}$. One says that the sequence $\left(X_{i}, x_{i}\right)_{i \in \mathbf{N}}$ converges to $\left(X_{\infty}, x_{\infty}\right)$ in the pointed Gromov-Hausdorff topology if for any $R>0, \mathrm{~d} \mathcal{E}_{\mathcal{H}}\left(B_{X_{i}}\left(x_{i}, R\right), B_{X_{\infty}}\left(x_{\infty}\right.\right.$, $R)) \rightarrow 0$ as $i \rightarrow+\infty$. (In fact, this definition holds only for length spaces, which will be sufficient in our situation.)

To deal with the Gromov-Hausdorff distance between $X_{1}$ and $X_{2}$, it is convenient to avoid the third space $Z$ by using $\varepsilon$-approximations between $X_{1}$ and $X_{2}$.

\section{Definition 4.2}

Given two metric spaces $X_{1}, X_{2}$ and $\varepsilon>0$, an $\varepsilon$-approximation (or $\varepsilon$-isometry) from $X_{1}$ to $X_{2}$ is a map $f: X_{1} \rightarrow X_{2}$ such that

(1) for any $x, x^{\prime} \in X_{1},\left|d_{X_{2}}\left(f(x), f\left(x^{\prime}\right)\right)-d_{X_{1}}\left(x, x^{\prime}\right)\right|<\varepsilon$;

(2) the $\varepsilon$-neighborhood of $f\left(X_{1}\right)$ is equal to $X_{2}$.

Then one can show (see [5, Corollary 7.3.28]) that $\mathrm{d} \mathscr{H} \mathscr{H}\left(X_{1}, X_{2}\right)<\varepsilon$ if there exists a $2 \varepsilon$-approximation from $X_{1}$ to $X_{2}$ and similarly an $\varepsilon$-approximation exists if $\mathrm{d} \mathscr{H} \mathscr{H}\left(X_{1}, X_{2}\right)<2 \varepsilon$. Let us insist on the fact that these approximations may be neither continuous nor even measurable.

Our goal is to prove the following.

PROPOSITION 4.3

Up to extraction and renumbering, the sequence $\left(Y_{k}, g_{k}, y_{k}\right)$ satisfies the following. 
(1) There exists a complete pointed length space $\left(Y_{\infty}, d_{\infty}, y_{\infty}\right)$ such that $\left(Y_{k}, g_{k}\right.$, $y_{k}$ ) converges in the pointed Gromov-Hausdorff topology to a metric space $\left(Y_{\infty}, d_{\infty}, y_{\infty}\right)$. Moreover, $\left(Y_{\infty}, d_{\infty}\right)$ has Hausdorff dimension equal to $n$.

(2) There exist sequences of positive numbers $\left.\varepsilon_{k} \rightarrow 0, \delta_{k} \rightarrow 0, c_{k} \in\right] h\left(g_{k}\right)$, $h\left(g_{k}\right)+\delta_{k}\left[, R_{k} \rightarrow+\infty\right.$, such that $\varepsilon_{k} \leq \varepsilon\left(R_{k}\right)$ and $\delta_{k} \leq \delta\left(R_{k}\right)$, where $\varepsilon(\cdot)$ and $\delta(\cdot)$ are given by Lemma 3.12, and such that the following holds. Let

$$
F_{c_{k}}:\left(Y_{k}, g_{k}\right) \rightarrow\left(X, g_{0}\right)
$$

be the natural map as defined in Section 2. Then $F_{c_{k}} \circ \psi_{k}$ converges uniformly on compact sets to a map

$$
F: Y_{\infty} \longrightarrow X
$$

which is 1-Lipschitz.

The proof is divided in two steps described in the following sections.

\section{Existence of the limit and its properties}

Under the curvature bound (3) and the local volume estimate (11), the sequence $\left(Y_{k}, g_{k}\right)$ is "noncollapsing" and part (1) of Proposition 4.3 is a straightforward application of the Gromov and Cheeger-Colding compactness theorem (see [8, Theorem 1.6]). Before proving point (2) of Proposition 4.3, let us describe some features of the convergence and of the limit space which will be used later.

The continuity of the volume under the (pointed) Gromov-Hausdorff convergence is crucial for our purposes. For $\ell>0$, note $\mathscr{H}^{\ell}$ the $\ell$-dimensional Hausdorff measure of a metric space (see [5, Definition 1.7.7]).

THEOREM 4.4 ([8, Theorem 5.9])

Let $p_{i} \in Y_{i}$, let $p_{\infty} \in Y_{\infty}$ be their limit, and let $R>0$. Then

$$
\lim _{i \rightarrow+\infty} \operatorname{vol}_{g_{i}}\left(B\left(p_{i}, R\right)\right)=\mathscr{H}^{n}\left(B\left(p_{\infty}, R\right)\right) .
$$

In particular, $Y_{\infty}$ satisfies the Bishop-Gromov inequalities (9) and the Bishop inequality. By definition, a tangent cone at $p \in Y_{\infty}$ is a complete pointed GromovHausdorff limit, $\left\{Y_{\infty, p}, d_{\infty}, p_{\infty}\right\}$ of a sequence of rescaled space, $\left\{\left(Y_{\infty}, r_{i}^{-1} d, p\right)\right\}$, where $\left\{r_{i}\right\}$ is a positive sequence such that $r_{i} \rightarrow 0$. Indeed, by [12, Proposition 5.2], every such sequence has a convergent subsequence, but the limit might depend on the choice of the subsequence. Notice that this notion is different from the one described in [5, Chapter 8] where the authors require that the limit be unique (does not depend on the subsequence). 


\section{Definition 4.5}

The regular set $\mathcal{R}$ consists of those points, $p \in Y_{\infty}$, such that every tangent cone at $p$ is isometric to $\mathbf{R}^{n}$. The complementary $\delta=Y_{\infty} \backslash \mathcal{R}$ is the singular set.

Let $B_{0}^{n}(1) \subset \mathbf{R}^{n}$ be the unit ball.

\section{Definition 4.6}

The $\varepsilon$-regular set $\mathcal{R}_{\varepsilon}$ consists of those points, $p \in Y_{\infty}$, such that every tangent cone, $\left(Y_{\infty, p}, p_{\infty}\right)$, satisfies $d_{G H}\left(B\left(p_{\infty}, 1\right), B_{0}^{n}(1)\right)<\varepsilon$. A point in $Y_{\infty} \backslash \mathcal{R}_{\varepsilon}=\wp_{\varepsilon}$ is called $\varepsilon$-singular.

THEOREM 4.7 ([8, Theorem 5.14])

There exists $\varepsilon_{n}>0$ such that for $\varepsilon \leq \varepsilon_{n}, \stackrel{\circ}{\mathcal{R}}_{\varepsilon}$ has a natural smooth manifold structure. Moreover, for this parameterization, the metric on $\stackrel{\circ}{\mathcal{R}}_{\varepsilon}$ is bi-Hölder equivalent to a smooth Riemannian metric. The exponent $\alpha(\varepsilon)$ in this bi-Hölder equivalence satisfies $\alpha(\varepsilon) \rightarrow 1$ as $\varepsilon \rightarrow 0$.

THEOREM 4.8 ([8, Theorem 6.1])

We have

$$
\mathscr{H}^{n-2}(\&)=0 .
$$

Remark 4.9

Clearly, $\mathcal{R}=\bigcap_{\varepsilon>0} \mathcal{R}_{\varepsilon}$. The sets $\mathcal{R}_{\varepsilon}, \mathcal{R}$ are not necessarily open. However, for any $\varepsilon>0$, there is some $\delta \in(0, \varepsilon)$ such that $\mathcal{R}_{\delta} \subset \stackrel{\circ}{\mathcal{R}}_{\varepsilon}$ (see [8, Appendix A.1.5]). In [9, Section 3], it is also proved that $\stackrel{\circ}{\mathcal{R}}_{\varepsilon}$ is path connected. This important fact will be used in the last part of this text.

We now study the density of the Hausdorff measure. A consequence of Bishop's inequality is that

$$
\limsup _{r \rightarrow 0} \frac{\mathscr{H}^{n}(B(p, r))}{\operatorname{vol}_{\mathbf{R}^{n}}(r)} \leq 1 .
$$

Definition 4.10

The density at $p$ of $Y_{\infty}$ is

$$
\theta(p):=\liminf _{r \rightarrow 0} \frac{\mathscr{H}^{n}(B(p, r))}{\operatorname{vol}_{\mathbf{R}^{n}}(r)} .
$$


A consequence of $[8$, A.1.5] is the existence of some positive function $\tau(\varepsilon)$, with $\tau(\varepsilon) \rightarrow 0$ as $\varepsilon \rightarrow 0$, such that for every $p \in \mathcal{R}_{\varepsilon}$,

$$
\theta(p)>1-\tau(\varepsilon)
$$

Conversely, there exists a positive function $\varepsilon(\tau)$, satisfying $\varepsilon(\tau) \rightarrow 0$ as $\tau \rightarrow 0$ and such that

$$
\theta(p) \geq 1-\tau \Longrightarrow p \in \mathcal{R}_{\varepsilon(\tau)}
$$

\section{Remark 4.11}

A point $p$ is regular if and only if $\theta(p)=1$. From now on, we consider $\varepsilon \leq \varepsilon_{0}$, where $\varepsilon_{0} \leq \varepsilon_{n}$ is sufficiently small so that $\tau\left(\varepsilon_{0}\right)<1 / 2$; the density is thus strictly greater than $1 / 2$ on $\mathcal{R}_{\varepsilon}$.

\section{Existence of the natural map at the limit}

Let us now prove (2) of Proposition 4.3.

\section{Proof}

For every $k \in \mathbf{N}$ and $c>h\left(g_{k}\right)$, there exists a natural map $F_{c}:\left(Y_{k}, g_{k}\right) \rightarrow\left(X, g_{0}\right)$, described in Section 2. We need to choose the values of $c$ for each $g_{k}$ in order that $F_{c}$ satisfies some good properties. One argues as follows.

Given $m \in \mathbf{N}^{*}$, one chooses positive numbers $\varepsilon_{m} \leq \varepsilon_{2}(m)$ and $\delta_{m} \leq \delta_{2}(m)$ sufficiently small such that $\kappa\left(\varepsilon_{m}, \delta_{m}, m\right) \leq \frac{1}{m}$, where $\delta_{2}, \varepsilon_{2}$ and $\kappa$ are given by Lemma 3.13. One then defines

$$
\alpha_{m}=\max \left\{\alpha_{1}\left(\varepsilon_{m}, \delta_{m}\right), \alpha_{2}\left(\varepsilon_{m}, \delta_{m}\right), \alpha_{3}\left(\varepsilon_{m}, \delta_{m}\right), \kappa\left(\varepsilon_{m}, \delta_{m}, m\right)\right\},
$$

where $\alpha_{1}, \alpha_{2}$ and $\alpha_{3}$ are defined in Lemmas 3.9, 3.10, and 3.11, respectively.

We check that $\alpha_{m} \rightarrow 0$ as $m \rightarrow+\infty$. By the hypothesis (73), there exists $k_{1}(m) \in$ $\mathbf{N}$ such that for any $k \geq k_{1}(m), \operatorname{vol}_{g_{k}}\left(Y_{k}\right) \leq\left(1+\varepsilon_{m}\right) \operatorname{vol}_{g_{0}}(X)$. Since for $m$ fixed $B_{g_{k}}\left(y_{k}, m\right)$ converges to $B_{\infty}\left(y_{\infty}, m\right)$, there exists $k_{2}(m) \in \mathbf{N}$ such that for any $k \geq$ $k_{2}(m)$, there exist $\alpha_{m}$-approximations from $B_{\infty}\left(y_{\infty}, m\right)$ to $B_{g_{k}}\left(y_{k}, m\right)$. Define $k(m):=\max \left\{k_{1}(m), k_{2}(m)\right\}$, and let $\psi_{m}: B_{\infty}\left(y_{\infty}, m\right) \longrightarrow B_{g_{k}}\left(y_{k(m)}, m\right)$ be an $\alpha_{m}$-approximation. One can assume that $\psi_{m}\left(y_{\infty}\right)=y_{k(m)}$. Choose $c_{m}$ such that $h\left(g_{k}\right)<c_{m}<h\left(g_{k}\right)+\delta_{m}$, and consider

$$
F_{c_{m}} \circ \psi_{m}: B_{\infty}\left(y_{\infty}, m\right) \longrightarrow X .
$$

Lemma 3.13 applies to $F_{c_{m}}$ on $B_{g_{k(m)}}\left(y_{k(m)}, m\right)$. Hence, for any $p, q \in B_{\infty}\left(y_{\infty}, m\right)$,

$$
\begin{aligned}
d_{g_{0}}\left(F_{c_{m}} \circ \psi_{m}(p), F_{c_{m}} \circ \psi_{m}(q)\right) & \leq\left(1+\alpha_{m}\right) d_{g_{k}}\left(\psi_{m}(p), \psi_{m}(q)\right)+\alpha_{m} \\
& \leq\left(1+\alpha_{m}\right) d_{\infty}(p, q)+\left(1+\alpha_{m}\right) \alpha_{m}+\alpha_{m}
\end{aligned}
$$


Applying the same reasoning as in Ascoli's theorem, one can show that for any compact $K \subset Y_{\infty}$, there exists a subsequence of $F_{c_{m}}$ converging to a map $F_{K}: K \rightarrow X$. We denote it by $F_{c_{\phi(m)}}$. If one uses an exhaustion of $Y_{\infty}$ by compact sets and a standard diagonal process, one can extract a subsequence of $F_{c_{\phi(m)}} \circ \psi_{\phi(m)}$ which converges uniformly on any compact set to a map $F: Y_{\infty} \rightarrow X$. It is easy to see that the map $F$ is 1-Lipschitz.

Then one renumbers the subsequences $Y_{k(\phi(m))}, \psi_{\phi(m)}$, and $F_{c_{\phi(m)}}$ such that, for any $m \in \mathbf{N}^{*}, \operatorname{vol}_{g_{m}}\left(Y_{m}\right) \leq\left(1+\varepsilon_{m}\right) \operatorname{vol}_{g_{0}}(X), h\left(g_{m}\right)<c_{m}<h\left(g_{m}\right)+\delta_{m}$, the inequalities of Lemmas 3.9, 3.10, and 3.11 hold with $\alpha_{1}, \alpha_{2}, \alpha_{3}$ replaced by $\alpha_{m}$, and those of Lemmas 3.12 and 3.13 hold on $B\left(y_{m}, m\right) \subset Y_{m}$ with $\kappa$ replaced by $\alpha_{m}$. For simplicity, the map $F_{c_{m}}$ will be denoted by $F_{m}$.

\section{The limit map $F: Y_{\infty} \longrightarrow X$ is isometric}

In this section we aim at proving that the limit map $F=\lim F_{k} \circ \psi_{k}$ is an isometry, that is, it is distance preserving. This will follow from the fact that $F$ is a volumepreserving 1-Lipschitz map. We prove first that $F$ preserves the volume.

\section{LEMMA 5.1}

Let $A \subset Y_{\infty}$ be a measurable subset. Then,

$$
\operatorname{vol}_{g_{0}}(F(A))=\mathscr{H}^{n}(A) \text {. }
$$

\section{Proof}

It suffices to prove the lemma when the set $A$ is an open ball. Indeed, let us assume that $F$ preserves the volume of balls, and let $A$ be a measurable set included in a ball $B:=B_{\infty}(p, r)$. Since $F$ is contracting it does not increase the volumes (see [16, Proposition 3.5]). Now, if $\operatorname{vol}_{g_{0}}(F(A))<\mathscr{H}^{n}(A)$ and since we have $\operatorname{vol}_{g_{0}}(F(B \backslash$ $A)) \leq \mathscr{H}^{n}(B \backslash A)$, we have a contradiction with the preservation of the volume of $B$. Similarly, if $A$ is a measurable set of finite measure we can apply the same argument with $A$ and $B \backslash A$ for any ball $B$.

It is then enough to prove that for every $B_{\infty}(p, r) \subset Y_{\infty}, \operatorname{vol}_{g_{0}}\left(F\left(B_{\infty}(p, r)\right)\right) \geq$ $\mathscr{H}^{n}\left(B_{\infty}(p, r)\right)$. By construction, $\overline{F\left(B_{\infty}(p, r)\right)}$ is the Hausdorff limit of $\overline{F_{k} \circ \psi_{k}\left(B_{\infty}(p, r)\right)}$.

We first show that this is also the Hausdorff limit of $\overline{F_{k}\left(B_{g_{k}}\left(\psi_{k}(p), r\right)\right)}$. Let $x \in$ $\overline{F\left(B_{\infty}(p, r)\right)}$ and $x_{k} \in F\left(B_{\infty}(p, r)\right)$ such that $x_{k} \rightarrow x$. Let $p_{k} \in B_{\infty}(p, r)$ such that $F\left(p_{k}\right)=x_{k}$. By definition of the $\alpha_{k}$-approximation, one has $d_{g_{k}}\left(\psi_{k}\left(p_{k}\right), \psi_{k}(p)\right)<$ $r+\alpha_{k}$. There exists $z_{k} \in B_{g_{k}}\left(\psi_{k}(p), r\right)$ such that $d_{g_{k}}\left(\psi_{k}\left(p_{k}\right), z_{k}\right)<\alpha_{k}$ (e.g., $z_{k}$ may be on the segment $\left.\left[\psi_{k}\left(p_{k}\right), \psi_{k}(p)\right]\right)$. Note that, by the triangular inequality, $d_{\infty}\left(p_{k}, y_{\infty}\right) \leq r+d_{\infty}\left(p, y_{\infty}\right)$, and recall that $\psi_{k}\left(y_{\infty}\right)=y_{g_{k}}$. Thus $\psi_{k}\left(p_{k}\right)$ remains 
at bounded distance from $y_{g_{k}}$. Then, applying Lemma 3.13 we have

$$
\begin{aligned}
d_{g_{0}}\left(F_{k}\left(z_{k}\right), F_{k}\left(\psi_{k}\left(p_{k}\right)\right)\right) & \leq\left(1+\alpha_{k}\right) d_{g_{k}}\left(z_{k}, \psi_{k}\left(p_{k}\right)\right)+\alpha_{k} \\
& \leq\left(1+\alpha_{k}\right) \alpha_{k}+\alpha_{k} \\
& \longrightarrow \rightarrow+\infty
\end{aligned}
$$

On the other hand, since $F_{k} \circ \psi_{k}$ converges uniformly to $F$ on compact sets, $F_{k}\left(\psi_{k}\left(p_{k}\right)\right)$ has the same limit as $F\left(p_{k}\right)=x_{k}$; that is, $F_{k}\left(\psi_{k}\left(p_{k}\right)\right) \rightarrow x$. From the inequality above one deduces that $F_{k}\left(z_{k}\right) \rightarrow x$, which shows that $x \in$ $\lim _{k \rightarrow \infty} \overline{F_{k}\left(B_{g_{k}}\left(\psi_{k}(p), r\right)\right)}$. One has then proved that $\overline{F\left(B_{\infty}(p, r)\right)} \subset$ $\lim _{k \rightarrow \infty} \overline{F_{k}\left(B_{g_{k}}\left(\psi_{k}(p), r\right)\right)}$. In order to prove the other inclusion, one argues similarly. Given $x \in \lim _{k \rightarrow \infty} \overline{F_{k}\left(B_{g_{k}}\left(\psi_{k}(p), r\right)\right)}$, there exists $x_{k} \in F_{k}\left(B_{g_{k}}\left(\psi_{k}(p), r\right)\right)$ such that $x_{k} \rightarrow x$, with $x_{k}=F_{k}\left(z_{k}\right)$ where $z_{k} \in B_{g_{k}}\left(\psi_{k}(p), r\right)$. As $\psi_{k}$ is an $\alpha_{k}$ approximation from $B_{\infty}\left(y_{\infty}, k\right)$ to $B\left(y_{g_{k}}, k\right)$, one has the inclusion $B_{g_{k}}\left(\psi_{k}(p), r\right) \subset$ $U_{\alpha_{k}} \psi_{k}\left(B_{\infty}\left(p, r+\alpha_{k}\right)\right)$ for large $k$, thus there exists $q_{k} \in B_{\infty}\left(p, r+\alpha_{k}\right)$ satisfying $d_{g_{k}}\left(z_{k}, \psi_{k}\left(q_{k}\right)\right)<\alpha_{k}$. As $Y_{\infty}$ is a length space, there exists $q_{k}^{\prime} \in B_{\infty}(p, r)$ such that $d_{\infty}\left(q_{k}^{\prime}, q_{k}\right)<\alpha_{k}$. Then $d_{g_{k}}\left(\psi_{k}\left(q_{k}^{\prime}\right), z_{k}\right) \leq d_{g_{k}}\left(\psi_{k}\left(q_{k}^{\prime}\right), \psi_{k}\left(q_{k}\right)\right)+d_{g_{k}}\left(\psi_{k}\left(q_{k}\right)\right.$, $\left.z_{k}\right)<3 \alpha_{k}$. Thus

$$
\begin{aligned}
d_{g_{0}}\left(F_{k} \circ \psi_{k}\left(q_{k}^{\prime}\right), x_{k}\right) & =d_{g_{0}}\left(F_{k} \circ \psi_{k}\left(q_{k}^{\prime}\right), F_{k}\left(z_{k}\right)\right) \\
& \leq\left(1+\alpha_{k}\right) d_{g_{k}}\left(\psi_{k}\left(q_{k}^{\prime}\right), z_{k}\right)+\alpha_{k} \\
& \leq\left(1+\alpha_{k}\right) 3 \alpha_{k}+\alpha_{k} \rightarrow 0 .
\end{aligned}
$$

Hence $d_{g_{0}}\left(F_{k} \circ \psi_{k}\left(q_{k}^{\prime}\right), x\right) \rightarrow 0$. As $F_{k} \circ \psi_{k}$ converges uniformly to $F$ on compact sets, one has $d_{g_{0}}\left(F\left(q_{k}^{\prime}\right), x\right) \rightarrow 0$, and thus $x \in \overline{F\left(B_{\infty}(p, r)\right)}$. This shows that $x \in$ $\overline{F\left(B_{\infty}(p, r)\right)}$ is the Hausdorff limit of $\overline{F_{k}\left(B_{g_{k}}\left(\psi_{k}(p), r\right)\right)}$.

In order to prove the lemma, it is then sufficient to prove that

$$
\begin{aligned}
\liminf _{k \rightarrow+\infty} \operatorname{vol}_{g_{0}}\left(\overline{F_{k}\left(B_{g_{k}}\left(\psi_{k}(p), r\right)\right)}\right) & \geq \liminf _{k \rightarrow+\infty} \operatorname{vol}_{g_{0}}\left(F_{k}\left(B_{g_{k}}\left(\psi_{k}(p), r\right)\right)\right) \\
& \geq \mathscr{H}^{n}\left(B_{\infty}(p, r)\right) .
\end{aligned}
$$

Indeed, inequality (80) will imply that

$$
\operatorname{vol}_{g_{0}}\left(F\left(\overline{B_{\infty}(p, r)}\right)\right) \geq \operatorname{vol}_{g_{0}}\left(\overline{F\left(B_{\infty}(p, r)\right)}\right) \geq \mathscr{H}^{n}\left(B_{\infty}(p, r)\right)
$$

and thus $\operatorname{vol}_{g_{0}}\left(F\left(B_{\infty}(p, r)\right)\right) \geq \mathscr{H}^{n}\left(B_{\infty}(p, r)\right)$ since $F$ being Lipschitz, we have

$$
\operatorname{vol}_{g_{0}}\left(F\left(\overline{B_{\infty}(p, r)}\right)\right)=\operatorname{vol}_{g_{0}}\left(F\left(B_{\infty}(p, r)\right)\right) .
$$

Recall that $N\left(F_{k}, x\right)$ is the number of preimages of $x$ by $F_{k}$. We denote by $X_{k, 1}$ the set of $x \in X$ such that $N\left(F_{k}, x\right)=1$. The construction of the sequence $\left(F_{k}\right)$, 
Lemma 3.10 and our choice of the $\alpha_{k}$ 's imply that $\operatorname{vol}_{g_{0}}\left(X_{k, 1}\right) \geq\left(1-\alpha_{k}\right) \operatorname{vol}_{g_{0}}(X)$ and

$$
\int_{X \backslash X_{k, 1}} N\left(F_{k}, x\right) \operatorname{dv}_{\mathrm{g}_{0}}(x) \leq \alpha_{k} \operatorname{vol}_{g_{0}}(X) .
$$

We also denote by $Y_{k, \alpha_{k}}$ the set of $y \in Y_{k}$ such that

$$
1-\alpha_{k} \leq\left|\operatorname{Jac} F_{k}(y)\right| \leq 1+\alpha_{k} .
$$

Then Lemma 3.9 implies that $\operatorname{vol}_{g_{k}}\left(Y_{k, \alpha_{k}}\right) \geq\left(1-\alpha_{k}\right) \operatorname{vol}_{g_{k}}\left(Y_{k}\right)$, for $k$ large enough. We then have

$$
\begin{aligned}
\operatorname{vol}_{g_{0}} & \left(F_{k}\left(B_{g_{k}}\left(\psi_{k}(p), r\right)\right)\right) \\
& =\int_{F_{k}\left(B_{g_{k}}\left(\psi_{k}(p), r\right)\right)} \mathrm{dv}_{\mathrm{g}_{0}} \\
& =\int_{F_{k}\left(B_{g_{k}}\left(\psi_{k}(p), r\right)\right) \cap X_{k, 1}} \mathrm{dv}_{\mathrm{g}_{0}}(x)+\operatorname{vol}_{g_{0}}\left(F_{k}\left(B_{g_{k}}\left(\psi_{k}(p), r\right)\right) \backslash X_{k, 1}\right) \\
& \geq \int_{B_{g_{k}}\left(\psi_{k}(p), r\right) \cap F_{k}^{-1}\left(X_{k, 1}\right) \cap Y_{k, \alpha_{k}}}\left|\mathrm{Jac} F_{k}(y)\right| \mathrm{dv}_{g_{k}}(y) \\
& \geq\left(1-\alpha_{k}\right) \operatorname{vol}_{g_{k}}\left(B_{g_{k}}\left(\psi_{k}(p), r\right) \cap F_{k}^{-1}\left(X_{k, 1}\right) \cap Y_{k, \alpha_{k}}\right) .
\end{aligned}
$$

On the other hand, using (82) and (81) we have

$$
\begin{aligned}
\operatorname{vol}_{g_{k}} & \left(F_{k}^{-1}\left(X \backslash X_{k, 1}\right) \cap Y_{k, \alpha_{k}}\right) \\
& \leq \int_{F_{k}^{-1}\left(X \backslash X_{k, 1}\right) \cap Y_{k, \alpha_{k}}} \frac{\left|\mathrm{Jac} F_{k}\right|}{1-\alpha_{k}} \mathrm{dv}_{g_{k}} \\
& \leq \frac{1}{1-\alpha_{k}} \int_{X \backslash X_{k, 1}} N\left(F_{k}, x\right) \mathrm{dv}_{\mathrm{g}_{0}}(x) \\
& \leq \frac{\alpha_{k}}{1-\alpha_{k}} \operatorname{vol}_{g_{0}}(X),
\end{aligned}
$$

and, consequently,

$$
\begin{aligned}
\operatorname{vol}_{g_{k}}( & \left.B_{g_{k}}\left(\psi_{k}(p), r\right) \cap F_{k}^{-1}\left(X_{k, 1}\right) \cap Y_{k, \alpha_{k}}\right) \\
= & \operatorname{vol}_{g_{k}}\left(B_{g_{k}}\left(\psi_{k}(p), r\right) \cap Y_{k, \alpha_{k}}\right) \\
\quad & \quad-\operatorname{vol}_{g_{k}}\left(B_{g_{k}}\left(\psi_{k}(p), r\right) \cap F_{k}^{-1}\left(X \backslash X_{k, 1}\right) \cap Y_{k, \alpha_{k}}\right) \\
\geq & \operatorname{vol}_{g_{k}}\left(B_{g_{k}}\left(\psi_{k}(p), r\right)\right)-\alpha_{k} \operatorname{vol}_{g_{k}}\left(Y_{k}\right)-\frac{\alpha_{k}}{1-\alpha_{k}} \operatorname{vol}_{g_{0}}(X) .
\end{aligned}
$$


Plugging this inequality in (83) one gets

$$
\begin{aligned}
\operatorname{vol}_{g_{0}}\left(F_{k}\left(B_{g_{k}}\left(\psi_{k}(p), r\right)\right)\right) \geq & \left(1-\alpha_{k}\right) \operatorname{vol}_{g_{k}}\left(B_{g_{k}}\left(\psi_{k}(p), r\right)\right) \\
& -\left(1-\alpha_{k}\right) \alpha_{k} \operatorname{vol}_{g_{k}}\left(Y_{k}\right)-\alpha_{k} \operatorname{vol}_{g_{0}}(X) .
\end{aligned}
$$

As $B_{g_{k}}\left(\psi_{k}(p), r\right)$ converges to $B_{\infty}(p, r)$ in the Gromov-Hausdorff topology, Theorem 4.4 implies that $\lim _{k \rightarrow \infty} \operatorname{vol}_{g_{k}}\left(B_{g_{k}}\left(\psi_{k}(p), r\right)\right)=\mathscr{H}^{n}\left(B_{\infty}(p, r)\right)$; hence

$$
\liminf _{k \rightarrow \infty} \operatorname{vol}_{g_{0}}\left(F_{k}\left(B_{g_{k}}\left(\psi_{k}(p), r\right)\right)\right) \geq \mathscr{H}^{n}\left(B_{\infty}(p, r)\right),
$$

which proves the lemma.

We now prove that $F$ is injective on the set of points where the density is larger than $1 / 2$.

\section{LEMMA 5.2}

The map $F$ is injective on $\mathcal{R}_{\varepsilon}$ for $\epsilon \leq \epsilon_{0}$.

\section{Proof}

Suppose that there are $p_{1}, p_{2} \in \mathcal{R}_{\varepsilon}$ such that $F\left(p_{1}\right)=F\left(p_{2}\right)$. As $F$ is 1-Lipschitz, we have for every $r>0$,

$$
F\left(B_{\infty}\left(p_{1}, r\right) \cup B_{\infty}\left(p_{2}, r\right)\right) \subset B_{g_{0}}\left(F\left(p_{1}\right), r\right) .
$$

By Lemma 5.1,

$$
\begin{aligned}
\mathscr{H}^{n}\left(B_{\infty}\left(p_{1}, r\right) \cup B_{\infty}\left(p_{2}, r\right)\right) & =\operatorname{vol}_{g_{0}}\left(F\left(B_{\infty}\left(p_{1}, r\right) \cup B_{\infty}\left(p_{2}, r\right)\right)\right) \\
& \leq \operatorname{vol}_{g_{0}}\left(B_{\infty}\left(F\left(p_{1}\right), r\right)\right) .
\end{aligned}
$$

For $r<d\left(p_{1}, p_{2}\right) / 2$, the balls $B_{\infty}\left(p_{1}, r\right)$ and $B_{\infty}\left(p_{2}, r\right)$ are disjoint. Hence, dividing (84) by $\operatorname{vol}_{\mathbf{R}^{n}}(r)$, we get

$$
\frac{\mathscr{H}^{n}\left(B_{\infty}\left(p_{1}, r\right)\right)}{\operatorname{vol}_{\mathbf{R}^{n}}(r)}+\frac{\mathscr{H}^{n}\left(B_{\infty}\left(p_{2}, r\right)\right)}{\operatorname{vol}_{\mathbf{R}^{n}}(r)} \leq \frac{\operatorname{vol}_{g_{0}}\left(B_{g_{0}}\left(F\left(p_{1}\right), r\right)\right)}{\operatorname{vol}_{\mathbf{R}^{n}}(r)} .
$$

Taking the liminf as $r \rightarrow 0$ yields

$$
\theta\left(p_{1}\right)+\theta\left(p_{2}\right) \leq \theta\left(F\left(p_{1}\right)\right)=1
$$

which is a contradiction, since $\theta>1 / 2$ on $\mathcal{R}_{\varepsilon}$ if $\varepsilon<\varepsilon_{0}$ (see Remark 4.11).

\section{LEMMA 5.3}

The map $F$ is open on $\stackrel{\circ}{\mathcal{R}}_{\varepsilon}$ for $\epsilon \leq \epsilon_{0}$. 


\section{Proof}

Let $p \in \stackrel{\circ}{R}_{\varepsilon}$. We have to prove that there exists $\eta>0$ such that $B_{g_{0}}(F(p), \eta) \subset$ $F\left(\stackrel{\circ}{\mathcal{R}}_{\varepsilon}\right)$. There exists $r>0$ such that $B_{\infty}(p, 2 r) \subset \stackrel{\circ}{\mathcal{R}}_{\varepsilon}$. For the sake of simplicity we shall note $B:=B_{\infty}(p, r)$. By the previous lemma, $F(p) \notin F(\partial B)$. Thus, by compactness of $\partial B$ and continuity of $F$, there exists $\eta>0$ such that $d_{g_{0}}(F(p), F(\partial B))>$ $\eta$. Notice that, since $F$ is 1 -Lipschitz, $\eta<r$. Here, one could use the theory of local degree as in [3, Appendix C]; however, $Y_{\infty}$ is not, a priori, a manifold and it may even be not locally Lipschitz equivalent to $\mathbf{R}^{n}$. Let $R>2 r+d_{\infty}\left(y_{\infty}, p\right)$ be a fixed radius; it satisfies $\psi_{k}\left(B_{\infty}(p, 2 r)\right) \subset B_{g_{k}}\left(y_{g_{k}}, R\right)$ for large $k$. Let $z_{k}=\psi_{k}(p)$ and $B_{k}:=B\left(z_{k}, r\right)$. The choice of $R$ and the fact that the $\psi_{k}$ 's are approximations show that $B_{k} \subset B\left(y_{g_{k}}, R\right)$, for $k$ large enough. We choose $k$ large enough such that $\mathrm{d}_{\mathscr{H}}\left(F_{k}\left(\partial B_{k}\right), F(\partial B)\right) \leq \frac{\eta}{10}$. This is possible since $\mathrm{d}_{\mathscr{H}}\left(\psi_{k}(\partial B), \partial B_{k}\right)$ goes to zero, $F_{k} \circ \psi_{k}$ converges to $F$, and $F(p)$ is at distance from $F(\partial B)$ larger than $\eta$. Let $C$ (resp., $e_{k}$ ) be the connected component of $X \backslash F(\partial B)$ (resp., $X \backslash F_{k}\left(\partial B_{k}\right)$ ), which contains $F(p)$ (resp., $F_{k}\left(z_{k}\right)$ ). Now the ball $B(F(p), \eta / 10)$ is included in $\varphi$, and for $k$ large enough, $B\left(F_{k}\left(z_{k}\right), \eta / 10\right)$ is included in $\varrho_{k}$. On the other hand, by [11, Corollary 4.1.26], $\operatorname{deg}\left(F_{k} \mid B_{k}\right)$ is constant on $\ell_{k}$, where, for a subset $A \subset Y_{k}$,

$$
\operatorname{deg}\left(F_{k} \mid A\right)(x)=\sum_{y \in F_{k}^{-1}(x) \cap A} \operatorname{sign~Jac~} F_{k}(y) .
$$

We show that $\operatorname{deg}\left(F_{k} \mid B_{k}\right)=1$ on $\ell_{k}$ as follows. We have to prove that at least one point in $\ell_{k}$ this degree is 1 since it is constant on this set. In order to do that, we shall show that the set of such points has positive measure. Denote again by $X_{k, 1} \subset X$ the set of $x \in X$ such that $N\left(F_{k}, x\right)=1$; that is, $x$ has one preimage by $F_{k}$. By Lemma 3.10, $\operatorname{vol}_{g_{0}}\left(X_{k, 1}\right) \geq\left(1-\alpha_{k}\right) \operatorname{vol}_{g_{0}}(X)$. The intersection of $X_{k, 1}$ with $\ell_{k}$ has a positive measure for $k$ large enough; indeed, $B\left(F_{k}\left(z_{k}\right), \frac{\eta}{10}\right) \subset \ell_{k}$ and its volume is bounded below by (11) and $\operatorname{vol}\left(B\left(F_{k}\left(z_{k}\right), \frac{\eta}{10}\right) \backslash X_{k, 1}\right) \longrightarrow 0$ as $k \rightarrow+\infty$. Now, by Lemma 3.12, one has $F_{k}\left(B\left(z_{k}, \frac{\eta}{20 \sqrt{n}}\right)\right) \subset B\left(F_{k}\left(z_{k}\right), \frac{\eta}{10}\right)$ and $B\left(z_{k}, \frac{\eta}{20 \sqrt{n}}\right) \subset B_{k}$ for large $k$, and an argument similar to the one used in (80) shows that the volume of the image is bounded below. It thus intersects $X_{k, 1}$ on a set of positive measure for $k$ large enough. This proves that $\operatorname{deg}\left(F_{k} \mid B_{k}\right)=1$ on $\ell_{k}$. Since $B\left(F_{k}\left(z_{k}\right), \eta / 10\right)$ converges to $B(F(p), \eta / 10)$, this last ball is included in $\ell_{k}$ for $k$ large; hence, any point in $B\left(F(p), \frac{\eta}{10}\right)$ has a preimage by $F_{k}$ in $B_{k}$. By taking the limit when $k$ goes to $+\infty$, we get $B\left(F(p), \frac{\eta}{10}\right) \subset F(\overline{B(p, r)}) \subset F(B(p, 2 r)) \subset F\left(\stackrel{\circ}{\mathcal{R}_{\varepsilon}}\right)$.

\section{LEMMA 5.4}

There exists $c(\varepsilon)>0$ such that $F: \stackrel{\circ}{\mathcal{R}}_{\varepsilon} \longrightarrow F\left(\stackrel{\circ}{\mathcal{R}}_{\varepsilon}\right) \subset X$ is locally $(1+c(\varepsilon))$-biLipschitz. Moreover, $c(\varepsilon) \rightarrow 0$ as $\varepsilon \rightarrow 0$. 


\section{Proof}

The idea is the following: we already know that $F$ is 1-Lipschitz and volume preserving. In particular, a ball $B_{\infty}(p, r) \subset Y_{\infty}$ is sent into a ball $B_{g_{0}}(F(p), r) \subset X$. If the ball in $Y_{\infty}$ is in the almost-regular part and has a small radius, its volume is close to the Euclidean one, and so is the volume of the hyperbolic ball. One can then estimate how much the image of $B_{\infty}(p, r)$ is close to filling $B_{g_{0}}(F(p), r)$. If one considers the images of two disjoint balls, one can estimate how the corresponding hyperbolic balls overlap, and thus the distance between their centers.

Let $p \in \stackrel{\circ}{R}_{\varepsilon}$. Let $r(p, \varepsilon)>0$ be a radius such that for every $0<r \leq r(p, \varepsilon)$,

$$
\frac{\mathscr{H}^{n}\left(B_{\infty}(p, r)\right)}{\operatorname{vol}_{\mathbf{R}^{n}}(r)} \geq 1-\tau(\varepsilon),
$$

and let $r_{\varepsilon}=\min \{\varepsilon, r(p, \varepsilon)\}$. One can assume that $r_{\varepsilon}$ is smaller than the injectivity radius of $X$. Let $0<r<r_{\varepsilon}^{2}$ be such that $B_{\infty}(p, r) \subset \mathcal{R}_{\varepsilon}$. For every $q \in B_{\infty}(p, r)$, $B_{\infty}\left(p, r_{\varepsilon}-r_{\varepsilon}^{2}\right) \subset B_{\infty}\left(q, r_{\varepsilon}\right)$. Thus,

$$
\begin{aligned}
\mathscr{H}^{n}\left(B_{\infty}\left(q, r_{\varepsilon}\right)\right) & \geq \mathscr{H}^{n}\left(B_{\infty}\left(p, r_{\varepsilon}-r_{\varepsilon}^{2}\right)\right) \\
& \geq(1-\tau(\varepsilon)) \operatorname{vol}_{\mathbf{R}^{n}}\left(r_{\varepsilon}-r_{\varepsilon}^{2}\right) \\
& \geq(1-\tau(\varepsilon))\left(1-r_{\varepsilon}\right)^{n} \operatorname{vol}_{\mathbf{R}^{n}}\left(r_{\varepsilon}\right) .
\end{aligned}
$$

Suppose that there exist $p_{1}, p_{2} \in B_{\infty}(p, r), p_{1} \neq p_{2}$, and a number $0<\rho<1$ such that

$$
d_{g_{0}}\left(F\left(p_{1}\right), F\left(p_{2}\right)\right) \leq \rho d_{\infty}\left(p_{1}, p_{2}\right)
$$

Define $r^{\prime}=d_{\infty}\left(p_{1}, p_{2}\right) / 2>0$, and notice that $r^{\prime}<r$. By (74) and the BishopGromov inequality (9), for $i=1,2$, one has

$$
\mathscr{H}^{n}\left(B_{\infty}\left(p_{i}, r^{\prime}\right)\right) \geq \mathscr{H}^{n}\left(B_{\infty}\left(p_{i}, r_{\varepsilon}\right)\right) \frac{\operatorname{vol}_{\mathbf{H}^{n}}\left(r^{\prime}\right)}{\operatorname{vol}_{\mathbf{H}^{n}}\left(r_{\varepsilon}\right)} .
$$

Thus, by Lemma 5.1, inequality (85) and Bishop-Gromov inequality we have

$$
\begin{aligned}
\operatorname{vol}_{g_{0}} & \left(F\left(B_{\infty}\left(p_{1}, r^{\prime}\right) \cup B_{\infty}\left(p_{2}, r^{\prime}\right)\right)\right) \\
& =\mathscr{H}^{n}\left(B_{\infty}\left(p_{1}, r^{\prime}\right)\right)+\mathscr{H}^{n}\left(B_{\infty}\left(p_{2}, r^{\prime}\right)\right) \\
& \geq 2(1-\tau(\varepsilon))\left(1-r_{\varepsilon}\right)^{n} \frac{\operatorname{vol}_{\mathbf{H}^{n}}\left(r^{\prime}\right)}{\operatorname{vol}_{\mathbf{H}^{n}}\left(r_{\varepsilon}\right)} \operatorname{vol}_{\mathbf{R}^{n}}\left(r_{\varepsilon}\right) \\
& \geq 2(1-\tau(\varepsilon))\left(1-r_{\varepsilon}\right)^{n} \frac{\operatorname{vol}_{\mathbf{R}^{n}}(\varepsilon)}{\operatorname{vol}_{\mathbf{H}^{n}}(\varepsilon)} \operatorname{vol}_{\mathbf{R}^{n}}\left(r^{\prime}\right) \\
& \geq 2 \vartheta(\varepsilon) \operatorname{vol}_{\mathbf{R}^{n}}\left(r^{\prime}\right)
\end{aligned}
$$

where $\vartheta(\varepsilon)=(1-\tau(\varepsilon))(1-\varepsilon)^{n} \frac{\operatorname{vol}_{\mathbf{R}^{n}}(\varepsilon)}{\operatorname{vol}_{\mathbf{H}^{n}}(\varepsilon)} \rightarrow 1$ as $\varepsilon \rightarrow 0$ 
On the other hand,

$$
F\left(B_{\infty}\left(p_{1}, r^{\prime}\right) \cup B_{\infty}\left(p_{2}, r^{\prime}\right)\right) \subset B_{g_{0}}\left(F\left(p_{1}\right), r^{\prime}\right) \cup B_{g_{0}}\left(F\left(p_{2}\right), r^{\prime}\right) .
$$

Hence

$$
\begin{aligned}
\operatorname{vol}_{g_{0}}( & \left.F\left(B_{\infty}\left(p_{1}, r^{\prime}\right) \cup B_{\infty}\left(p_{2}, r^{\prime}\right)\right)\right) \\
\leq & \operatorname{vol}_{g_{0}}\left(B_{g_{0}}\left(F\left(p_{1}\right), r^{\prime}\right)\right)+\operatorname{vol}_{g_{0}}\left(B_{g_{0}}\left(F\left(p_{2}\right), r^{\prime}\right)\right) \\
& -\operatorname{vol}_{g_{0}}\left(B_{g_{0}}\left(F\left(p_{1}\right), r^{\prime}\right) \cap B_{g_{0}}\left(F\left(p_{2}\right), r^{\prime}\right)\right) .
\end{aligned}
$$

For any $x \in X$ and any $s>0$ smaller than the injectivity radius of $X$ one has $\operatorname{vol}_{g_{0}}(B(x, s))=\operatorname{vol}_{\mathbf{H}^{n}}(s)$. Let $x$ be the middle point of the segment $\left[F\left(p_{1}\right) F\left(p_{2}\right)\right]$. Then

$$
B\left(x, r^{\prime}(1-\rho)\right) \subset B\left(F\left(p_{1}\right), r^{\prime}\right) \cap B\left(F\left(p_{2}\right), r^{\prime}\right) .
$$

Indeed, if $x^{\prime} \in B\left(x, r^{\prime}(1-\rho)\right)$, then $d\left(x^{\prime}, F\left(p_{i}\right)\right) \leq d\left(x^{\prime}, x\right)+d\left(x, F\left(p_{i}\right)\right)<r^{\prime}(1-$ $\rho)+\rho r^{\prime}=r^{\prime}$ for $i=1,2$. Thus (92) gives

$$
\begin{aligned}
\operatorname{vol}_{g_{0}} & \left(F\left(B\left(p_{1}, r^{\prime}\right) \cup B\left(p_{2}, r^{\prime}\right)\right)\right) \\
\leq & 2 \operatorname{vol}_{\mathbf{H}^{n}}\left(r^{\prime}\right)-\operatorname{vol}_{\mathbf{H}^{n}}\left(r^{\prime}(1-\rho)\right) \\
\leq & 2 \operatorname{vol}_{\mathbf{R}^{n}}\left(r^{\prime}\right) \frac{\operatorname{vol}_{\mathbf{H}^{n}}\left(r^{\prime}\right)}{\operatorname{vol}_{\mathbf{R}^{n}}\left(r^{\prime}\right)}-(1-\rho)^{n} \operatorname{vol}_{\mathbf{R}^{n}}\left(r^{\prime}\right) \\
\leq & 2 \operatorname{vol}_{\mathbf{R}^{n}}\left(r^{\prime}\right) \frac{\operatorname{vol}_{\mathbf{H}^{n}}(\varepsilon)}{\operatorname{vol}_{\mathbf{R}^{n}}(\varepsilon)}-(1-\rho)^{n} \operatorname{vol}_{\mathbf{R}^{n}}\left(r^{\prime}\right) \\
& =\left(2 \frac{\operatorname{vol}_{\mathbf{H}^{n}}(\varepsilon)}{\operatorname{vol}_{\mathbf{R}^{n}}(\varepsilon)}-(1-\rho)^{n}\right) \operatorname{vol}_{\mathbf{R}^{n}}\left(r^{\prime}\right) .
\end{aligned}
$$

For the third inequality we have used Bishop-Gromov's inequality. From (91) and (96), we find

$$
(1-\rho)^{n} \leq 2\left(\frac{\operatorname{vol}_{\mathbf{H}^{n}}(\varepsilon)}{\operatorname{vol}_{\mathbf{R}^{n}}(\varepsilon)}-\vartheta(\varepsilon)\right) \rightarrow 0 .
$$

Therefore,

$$
\rho \geq 1-2^{1 / n}\left(\frac{\operatorname{vol}_{\mathbf{H}^{n}}(\varepsilon)}{\operatorname{vol}_{\mathbf{R}^{n}}(\varepsilon)}-\vartheta(\varepsilon)\right)^{1 / n}:=1-c(\varepsilon) \rightarrow 1,
$$

as $\varepsilon \rightarrow 0$. One has proved that inside the ball $B(p, r)$,

$$
d_{g_{0}}\left(F\left(p_{1}\right), F\left(p_{2}\right)\right) \geq\left(1-c_{1}(\varepsilon)\right) d_{\infty}\left(p_{1}, p_{2}\right),
$$

and the proof of the lemma follows by choosing $c(\varepsilon)$ so that $1-c_{1}(\varepsilon) \geq(1+c(\varepsilon))^{-1}$. 


\section{Remark 5.5}

On the connected (see Remark 4.9) open set $F\left(\stackrel{\circ}{\mathcal{R}}_{\varepsilon}\right) \subset X$, the metric $g_{0}$ induces a distance $\rho_{\varepsilon}$. The above lemma shows that $F:\left(\stackrel{\circ}{\mathcal{R}}_{\varepsilon}, d_{\infty}\right) \longrightarrow\left(F\left(\stackrel{\circ}{\mathcal{R}_{\varepsilon}}\right), \rho_{\varepsilon}\right)$ is a $(1+$ $c(\varepsilon)$ )-bi-Lipschitz homeomorphism. If one can prove that $\rho_{\varepsilon}=d_{g_{0}}$, one deduces that $\mathcal{R}_{\varepsilon}$ has bounded diameter. One then concludes that $\mathrm{d} \mathscr{\mathscr { H }}\left(Y_{k}, Y_{\infty}\right) \rightarrow 0$ and that $F$ : $Y_{\infty} \rightarrow X$ is isometric.

More precisely, we prove the following proposition.

\section{PROPOSITION 5.6}

The set $F\left(\stackrel{\circ}{\mathcal{R}}_{\varepsilon}\right)$ satisfies the following:

(1) for any $x_{1}, x_{2} \in F\left(\stackrel{\circ}{\mathcal{R}}_{\varepsilon}\right), d_{g_{0}}\left(x_{1}, x_{2}\right)=\rho_{\varepsilon}\left(x_{1}, x_{2}\right)$;

(2) $F\left(\stackrel{\circ}{\varepsilon}_{\varepsilon}\right)=X$;

(3) $\quad F:\left(Y_{\infty}, d_{\infty}\right) \longrightarrow\left(X, d_{g_{0}}\right)$ is an isometry.

\section{Proof}

Let $x_{1}, x_{2} \in F\left(\stackrel{\circ}{\mathcal{R}}_{\varepsilon}\right)$. Without loss of generality, one can suppose that $x_{2}$ is not in the image of the cut-locus of $x_{1}$. Clearly, $\rho_{\varepsilon}\left(x_{1}, x_{2}\right) \geq d_{g_{0}}\left(x_{1}, x_{2}\right)$. Let $\gamma:[0,1] \longrightarrow X$ be a $g_{0}$-minimal geodesic from $x_{1}$ to $x_{2}$. We do not know that $\gamma$ is in $F\left(\stackrel{\circ}{R}_{\varepsilon}\right)$; we then prove that there exist paths in $F\left(\stackrel{\circ}{\mathscr{R}}_{\varepsilon}\right)$ arbitrarily close to $\gamma$. Let $r>0$ be a radius such that $B_{g_{0}}\left(x_{2}, r\right) \subset F\left(\stackrel{\circ}{\mathcal{R}}_{\varepsilon}\right)$. We consider geodesics with the origin $x_{1}$ and the extremity in $B\left(x_{2}, \delta\right)$, for a small $\delta>0$. More precisely, let $u=\dot{\gamma}(0)$; then for any $v \in U_{x_{1}} X$ such that $u \perp v$, one defines $\gamma_{s, v}(t)=\exp _{x_{1}}\left(t(u+s . v) d\left(x_{1}, x_{2}\right)\right)$. There exists $r(\delta)>0$ such that $\gamma_{s, v}(1) \in B\left(x_{2}, \delta\right)$ if $|s| \leq r(\delta)$ and one can choose $r(\delta) \rightarrow 0$ as $\delta$ goes to 0 .

We claim that for every $\delta>0$, there exists such a $\gamma_{s, v}$ which is embedded in $F\left(\stackrel{\circ}{R}_{\varepsilon}\right)$.

Let us show that one can find such $\gamma_{s, v}$ disjoint from $F(8)$, where $\delta$ is the singular set of $Y_{\infty}$ introduced in Definition 4.5. The idea is that if any $\gamma_{s, v}$ would hit $F(\&)$ at least in one point, then the Hausdorff dimension of $F(\&)$ would be larger than $n-1$, which is a contradiction. More precisely, one considers a truncated cone $U_{\delta}$ defined as follows. Let

$$
\Gamma:] 0, r(\delta)] \times\left(U_{x_{1}} X \cap u^{\perp}\right) \times[0,1] \rightarrow X
$$

be defined by $\Gamma(s, v, t)=\gamma_{s, v}(t)$. If $\delta$ is sufficiently small, then $\Gamma$ is an embedding. One defines $\left.\left.U_{\delta}=\Gamma(] 0, r(\delta)\right] \times\left(U_{x_{1}} X \cap u^{\perp}\right) \times[0,1]\right)$. Let us denote by $U_{\delta}(1 / 2)$ the 
hypersurface in $U_{\delta}$ defined as $\left.\left.\left.\Gamma(] 0, r(\delta)\right]\right) \times\left(U_{x_{1}} X \cap u^{\perp}\right) \times\{1 / 2\}\right)$ :

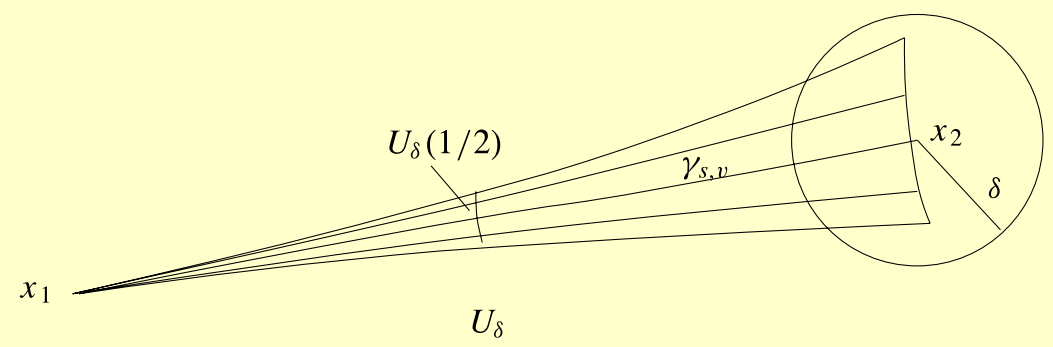

Let $P: U_{\delta} \rightarrow U_{\delta}(1 / 2)$ be the projection along geodesics defined by $P\left(\gamma_{s, v}(t)\right)=$ $\gamma_{s, v}(1 / 2)$. Since we are on a fixed Riemannian manifold, there exists a constant $C>0$ such that $P$ is $C$-Lipschitz from $U_{\delta}$ to $X$. In particular, $P$ decreases the Hausdorff dimension; that is,

$$
\begin{aligned}
\operatorname{dim}_{\mathscr{H}}\left(P\left(U_{\delta} \cap F(\delta)\right)\right) & \leq \operatorname{dim}_{\mathscr{H}}\left(U_{\delta} \cap F(\delta)\right) \\
& \leq \operatorname{dim}_{\mathscr{H}}(\delta) \\
& \leq n-2 \\
& <\operatorname{dim} U_{\delta}(1 / 2)=n-1 .
\end{aligned}
$$

Hence, there exists $x \in U_{\delta}(1 / 2)$ such that $x \notin \Pi(F(8))$. This implies that the geodesic $\gamma_{s, v}$ such that $x=\gamma_{s, v}(1 / 2)$ does not intersect $F(\&)$.

We now prove that $\gamma_{s, v}$ is embedded in $F\left(\stackrel{\circ}{\mathcal{R}}_{\varepsilon}\right)$. Let $t_{0} \in(0,1]$ be maximal such that $\gamma_{s, v}\left(\left[0, t_{0}[) \subset F\left(\stackrel{\circ}{\mathcal{R}}_{\varepsilon}\right)\right.\right.$. By Lemma 5.4 , the path $\beta=F^{-1} \circ \gamma_{s, v}$ is well defined on $\left[0, t_{0}\right.$ [ and has a length bounded by $(1+c(\varepsilon)) d\left(x_{1}, x_{2}\right)$. Since $F$ is bi-Lipschitz, $d_{g_{k}}\left(\beta(t), \beta\left(t^{\prime}\right)\right) \geq C\left|t^{\prime}-t\right|$, and hence there exists a limit $p=\lim _{t \rightarrow t_{0}} \beta(t) \in Y_{\infty}$. By continuity of $F, F(p)=\gamma_{s, v}\left(t_{0}\right)$, and since $\gamma_{s, v}\left(t_{0}\right) \notin F(8)$, we have that $p \notin$ \&. This implies that $p \in \mathcal{R}=\bigcap_{\varepsilon} \mathcal{R}_{\varepsilon}=\bigcap_{\varepsilon>0} \stackrel{\circ}{\mathcal{R}}_{\varepsilon}$ and, consequently, that $t_{0}=1$, because $\stackrel{\circ}{\mathcal{R}}_{\varepsilon}$ is open.

Hence

$$
\begin{aligned}
\rho_{\varepsilon}\left(x_{1}, x_{2}\right) & \leq \ell\left(\gamma_{s, v}\right)+d_{0}\left(\gamma_{s, v}(1), x_{2}\right) \\
& \leq \sqrt{1+r^{2}(\delta)} d_{0}\left(x_{1}, x_{2}\right)+\delta .
\end{aligned}
$$

As $\delta$ was arbitrary, this gives $\rho_{\varepsilon}\left(x_{1}, x_{2}\right) \leq d_{0}\left(x_{1}, x_{2}\right)$.

The second assertion is proved in a similar way. Suppose there is a ball $B(x, r) \subset$ $X \backslash F\left(\stackrel{\circ}{\mathscr{R}_{\varepsilon}}\right)$, and consider a geodesic $\gamma$ from a point $x_{1}$ inside $F\left(\stackrel{\circ}{\stackrel{\circ}{R}_{\varepsilon}}\right)$ to $x$. Then we 
find another geodesic from $x_{1}$, close to $\gamma$, disjoint from $F(\&)$, and with extremity in $X \backslash F\left(\stackrel{\circ}{\mathcal{R}}_{\varepsilon}\right)$. Arguing as above, we find a contradiction.

Now part (3) of Proposition 5.6 is straightforward. Using the density of $\stackrel{\circ}{\mathcal{R}}_{\varepsilon}$ in $Y_{\infty}$ and of $F\left(\stackrel{\circ}{\mathcal{R}}_{\varepsilon}\right)$ in $X$, we find that $F:\left(Y_{\infty}, d_{\infty}\right) \longrightarrow\left(X, d_{0}\right)$ is a $(1+c(\varepsilon))$-biLipschitz homeomorphism for any $0<\varepsilon<\varepsilon_{0}$, and thus is isometric.

\section{End of Proof of Theorem 1.3}

Proposition 5.6 implies that the diameter of $\left(Y, g_{k}\right)$ remains bounded. Thus, $\mathrm{d} \mathscr{F} H((Y$, $\left.\left.g_{k}\right),\left(Y_{\infty}, d_{\infty}\right)\right) \rightarrow 0$ (for the nonpointed convergence). As $\left(Y_{\infty}, d_{\infty}\right)$ is isometric to $\left(X, g_{0}\right)$, one deduces that $\mathrm{d} \mathscr{\mathscr { H }}\left(\left(Y, g_{k}\right),\left(X, g_{0}\right)\right) \rightarrow 0$ as $k \rightarrow \infty$. By [8, Theorem A.1.12], $Y$ is diffeomorphic to $X$. The fact that $f$ is homotopic to a diffeomorphism is classic for hyperbolic manifolds.

\section{References}

[1] M. ANDERSON, Canonical metrics on 3-manifolds and 4-manifolds, Asian J. Math. 10 (2006), 127-163. MR 2213687 (33)

[2] L. BESSIÈRES, Un théorème de rigidité différentielle, Comment. Math. Helv. 73 (1998), 443-479. MR 1633371 (30, 32)

[3] G. BESSON, G. COURTOIS, and S. GALLOT, Entropies et rigidités des espaces localement symétriques de courbure strictement négative, Geom. Funct. Anal. 5 (1995), 731-799. MR 1354289 (29, 31, 32, 33, 34, 35, 36, 38, 39, 61)

[4] - Minimal entropy and Mostow's rigidity theorems, Ergodic Theory Dynam. Systems 16 (1996), 623-649. MR 1406425 (34)

[5] D. BURAGO, Y. BURAGO, and S. IVANOV, A Course in Metric Geometry, Grad. Stud.

Math. 33, Amer. Math. Soc., Providence, 2001. MR 1835418 (53, 54)

[6] Y. BURAGO and V. ZALGALLER, Geometric Inequalities, Grundlehren Math. Wiss.

285, Springer, Berlin, 1988. MR 0936419 (31)

[7] J. CHEEGER and T. COLDING, Lower bounds on Ricci curvature and the almost rigidity of warped products, Ann. of Math. (2) 144 (1996), 189-237. MR 1405949 (49, 50)

[8] - On the structure of spaces with Ricci curvature bounded below, I, J. Diff. Geom. 46 (1997), 406-480. MR 1484888 (54, 55, 56, 66)

[9] - On the structure of spaces with Ricci curvature bounded below, II, J. Diff. Geom. 54 (2000), 13-35. MR 1815410 (55)

[10] F. T. FARRELL and L. E. JONES, Negatively curved manifolds with exotic smooth structures, J. Amer. Math. Soc. 2 (1989), 899-908. MR 1002632 (30)

[11] H. FEDERER, Geometric Measure Theory, Grundlehren Math. Wiss. 153, Springer,

New York, 1969. MR 0257325 (53, 61)

[12] M. GROMOV, Structures métriques pour les variétés riemanniennes, Textes

Mathématiques 1, CEDIC, Paris, 1981. MR 0682063 (54) 
[13] - Volume and bounded cohomology, Inst. Hautes Études Sci. Publ. Math. 56 (1982), 5-99. MR 0686042 (34)

[14] - Metric Structures for Riemannian and Non-Riemannian Spaces, Birkhäuser, Boston, 2007. MR 2307192 (53)

[15] O. KOBAYASHI, Scalar curvature of a metric with unit volume, Math. Ann. 279 (1987), 253-265. MR 0919505 (33)

[16] F. MORGAN, Geometric Measure Theory: A Beginner's Guide, 3rd ed. Academic Press, San Diego, 2000. MR 1775760 (42, 57)

\section{Bessières}

UJF-Grenoble 1/CNRS, Institut Fourier, UMR 5582, Grenoble F-38401, France;

laurent.bessieres@ujf-grenoble.fr

Besson

UJF-Grenoble 1/CNRS, Institut Fourier, UMR 5582, Grenoble F-38401, France;

G.Besson@ujf-grenoble.fr

\section{Courtois}

Institut de Mathématiques de Jussieu, Université de Paris VI, UMR 7586, 75252 Paris CEDEX 09, France; courtois@math.jussieu.fr

\section{Gallot}

UJF-Grenoble 1/CNRS, Institut Fourier, UMR 5582, Grenoble F-38401, France;

Sylvestre.Gallot@ujf-grenoble.fr 